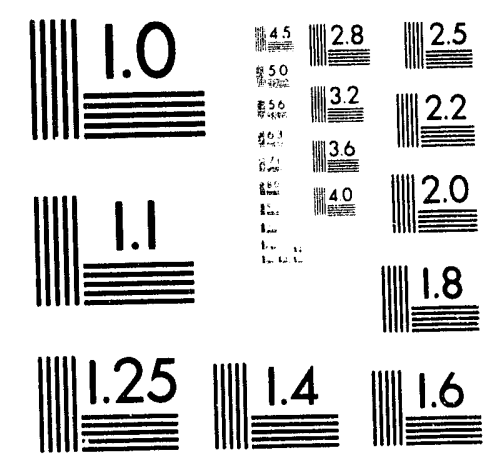



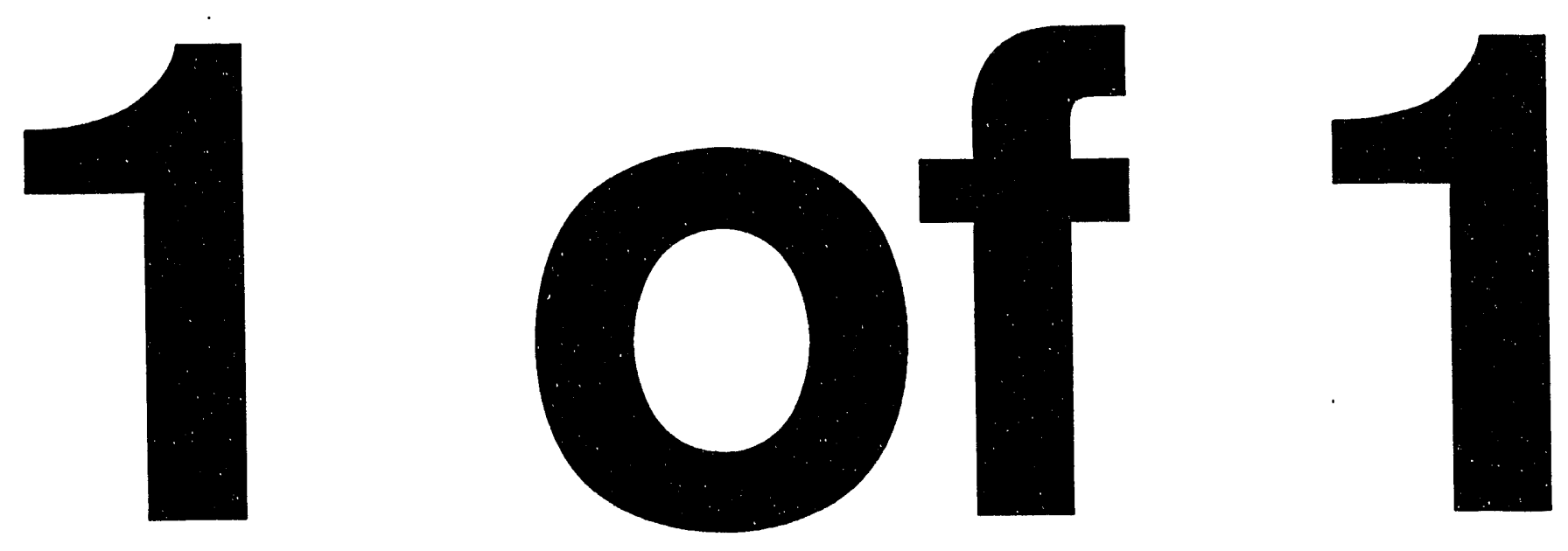
UCRL-LR-114182

Distribution Category UC-403

\title{
Two-Point Correlation Functions to Characterize Microgeometry and Estimate Permeabilities of Synthetic and Natural Sandstones
}

\author{
Stephen C. Blair \\ Patricia A. Berge \\ James G. Berryman
}

Manuscript date: August 1993

LAWRENCE LIVERMORE NATIONAL LABORATORY University of California - Livermore, California • 94551
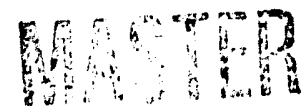


\title{
Two-Point Correlation Functions to Characterize Microgeometry and Estimate Permeabilities of Synthetic and Natural Sandstones
}

\begin{abstract}
We have developed an image-processing method for characterizing the microstructure of rock and other porous materials, and for providing a quantitative means for understanding the dependence of physical properties on the pore structure. This method is based upon the statistical properties of the microgeometry as observed in scanning electron micrograph (SEM) images of cross sections of porous materials. The method utilizes a simple statistical function, called the spatial correlation function, which can be used to predict bounds on permeability and other physical properties. We obtain estimates of the porosity and specific surface area of the material from the two-point correlation function. The specific surface area can be related to the permeability of porous materials using a Kozeny-Carman relation, and we show that the specific surface area measured on images of sandstones is consistent with the specific surface area used in a simple flow model for computation of permeability. In this paper, we discuss the two-point spatial correlation function and its use in characterizing microstructure features such as pore and grain sizes. We present estimates of permeabilities found using SEM images of several different synthetic and natural sandstones. Comparison of the estimates to laboratory measurements shows good agreement. Finally, we briefly discuss extension of this technique to two-phase flow.
\end{abstract}

\section{Introduction}

The physical and mechanical properties of rock have long been known to be strongly dependent on porosity. Moreover, studies of rock properties have shown that the geometry of the pore space plays a key role in determining the physical and mechanical properties. The development of digital image-processing techniques provides an effective tool for characterizing the microgeometry of the pore space in rocks and facilitates the systematic study of the relation of pore geometry to the rock properties of interest.

During the past few years, a variety of approaches have been used to relate microstructural information to the physical and mechanical or transport properties of the rock.
For instance, morphological analysis has been used to determine the shapes and sizes of the pores. Ehrlich et al. [1984] and Wissler [1987] among others have correlated morphological parameters measured on images of thin sections with laboratory measurenents of rock properties. These analyses provide useful insight into which aspects of the microgeometry are dominating the rock properties. Morphological analysis has also been used to provide input into effective medium models for material behavior. Koplik et al. [1984] and Doyen [1988] have used information such as pore throat size distribution in conjunction with effective medium theory to estimate permeability and conductivity. Fractal analysis of the pore 
geometry measured from images has also been used by Thompson et al. [1987] and Adler [1992]. Most of these studies have involved twodimensional analysis. However, Koplik et al. [1984] used serial sectioning techniques to reconstruct a three-dimensional image of pore space in a sandstone and obtained parameters for use in an effective medium analysis of rock properties.

Another approach is to use spatial correlation functions to characterize the microgeometry in a statistical way. Spatial correlation functions are commonly used in statistical physics to estimate physical properties of ideal composite materials. Corson $[1974 a, b]$ and Stanke [1986] formulated correlation functions for two-phase solids, and Berryman [1985b] developed a method to calculate the functions in a manner compatible with digital image processing. Torquato and Stell [1982], Berryman [1985a], and Berryman and Milton [1985, 1988] have used spatial correlation functions to estimate bounds on elastic and fluid transport properties of multicomponent porous materials. In many of these analyses, the material to be analyzed is formulated as an idealized two-phase material.

Our purpose is to demonstrate the use of spatial correlation functions as a tool to predict properties of geologic materials. As mentioned above, the theoretical basis of correlation functions is well developed and we want to explore the application of this theoretical machinery to the study of microstructure of heterogeneous materials such as rock. In this paper we provide a solid basis for using the two-point spatial correlation function in the study of cross sections of porous rocks such as sandstones. In the future we hope the method will be extended to the use of three-point correlation functions to estimate rock properties such as elastic constants and tortuosity.

In this paper we describe the spatial correlation function and discuss its usefulness in characterizing rock microstructure. We apply this approach to analysis of rock microstructure by assuming the rock is a two-phase material consisting only of grains and pores. We then use the spatial correlation function to characterize the microstructure of the pore-grain system. Moreover, we discuss compatibility of parameters measured on images with the Kozeny-Carman model [Carman, 1956; Dullien, 1979] for flow in porous media. We use the twopoint correlation function to characterize suites of samples of both synthetic and natural sandstones. The synthetic sandstones were formed from sintered glass beads and serve to illustrate the behavior of correlation functions for simple materials with porosities in the range of about 15 to $40 \%$. The natural sandstones we studied had porosities of about 10 to $30 \%$. We use parameters measured on scanning electron microscope (SEM) images to estimate porosity, effective pore and grain size, and specific surface area. We then estimate permeability for the samples and compare our estimates with laboratory measirements. We also present a brief discussion (if application of these correlation furictions to the study of multiphase flow.

\section{Methods}

\section{Analytical Methods}

\section{Spatial correlation functions}

A porous material such as rock can be idealized as a two-phase medium consisting of grains and pores. An elementary spatial correlation function that can be used to study this medium is the indicator function $f$ defined for any position $\mathbf{x}$ in the material by $f(\mathbf{x})=1$ if $\mathbf{x}$ is in the pore space and $f(\mathbf{x})=0$ if $\mathbf{x}$ is in a grain. If we take a cross section through the material and form a binary image of the cross section where the pore space is represented by 1 's and the grains are represented by 0 's, the image itself represents $f$. We can also see that if the medium is isotropic, the sum of $f$ over the area of the image of any cross section provides an estimate of the porosity $\phi$. This sum is the onepoint correlation function $S_{1}$.

A more complicated function is the twopoint correlation function $S_{2}$. For our isotropic two-component material, we define $S_{2}$ as the probability that two points separated by a 
distance $r$ will both be in the pore space. $S_{2}$ is a two-dimensional autocorrelation function and both $S_{1}$ and $S_{2}$ can be defined in terms of the indicator function $f(\mathbf{x})$ :

$$
\begin{gathered}
S_{1}=\langle f(x)\rangle=\phi \\
\text { and } \\
S_{2}(\mathbf{r})=\langle f(\mathbf{x}) f(\mathbf{x}+\mathbf{r})\rangle,
\end{gathered}
$$

where the brackets \langle\rangle denote volume averaging over all positions $x$. For an isotropic material, $S_{2}$ depends only on the distance $r=|r|$; thus we write $S_{2}(r)$. Both the one- and two-point correlation functions can be computed from a twodimensional image of a material. Berryman [1985b] presents an algorithm that computes $S_{2}$ by analyzing a digitized version of the image, comparing pairs of pixels at different separation distances and incrementing a sum when both pixels are in the pore space. More detailed discussions of the two-point correlation function and its properties can be found in Berryman [1985b] and Berryman and Blair [1986]. The twopoint correlation function $S_{2}(r)$ is of interest because it provides a measure of several important parameters of the microstructure in a very compact form, and its usefulness is not limited by any assumptions about particle shape.

Of particular note is the fact that the slope of the two-point correlation function near the origin is proportional to the specific surface area $s$ of the material:

$$
S_{2}^{\prime}(0)=-\frac{s}{4}
$$

[Debye et al., 1957; Berryman and Blair, 1986; Berryman, 1987], where the specific surface area is ciefined as the ratio of the total surface area of the pore-grain interface to the total volume of the porous material. This result follows from the definition of the two-point correlation function as the volume-weighted product of $f(\mathbf{x})$ and $f(x+r)$ integrated over the total volume of the porous material. This can be intuitively understood by considering the behavior of $f(\mathbf{x}) f(\mathbf{x}+\mathbf{r})$ at very small values of $\mathbf{r}$. If the point $x$ is in the central region of either a grain or a pore, the product $f(\mathbf{x}) f(\mathbf{x}+\mathbf{r})$ will be either zero or one and there will be no change in the product as $\mathbf{r}$ is increased slightly. However, if $\mathbf{x}$ is in the pore space but near the pore-grain interface, the product will change abruptly from one to zero as $r$ crosses the interface. To show this mathematically, we write $S_{2}$ as a volume integral in spherical coordinates and use the divergerce theorem of Gauss to transform the expression for its radial derivative into a surface integral over the material's total surface area. The limit for vanishing $r$ then yields Eq. (3). A detailed derivation can be found in Berryman [1987]. We want to emphasize that Eq. (3) is true for any porous material, regardless of particle shapes. In general, a synthetic material with very smooth grains would have a much lower specific surface area and, thus, a much flatter slope for $S_{2}$ than would be found for a natural material with rough grains and higher surface area.

\section{Examples based on idealized sphere packs}

To illustrate the application of $S_{2}$, we will first consider isotropic porous materials having spherical grains. The methods described are not limited to spherical particles, but spherical grains do make convenient examples of the use of these methods. For a random distribution of idealized fully penetrable spheres having radius $R$, the exact solution for $S_{2}(r)$ is known to be given by

$$
S_{2}(r)=\phi^{\alpha},
$$

where $\alpha=2$ for $r \geq 2 R$ and

$$
\alpha(r)=1+\frac{3 r}{4 R}-\frac{r^{3}}{16 R^{3}}
$$

for $\mathrm{r} \leq 2 \mathrm{R}$ [Weissberg, 1963; Torquato and Stell, 1983]. For the more realistic impenetrable (hard) sphere model, the radial particle distribution must be specified before $S_{2}(r)$ can be written in closed form [Torquato and Stell, 1982, 1985]. Values of $S_{2}$ for different porosities have been tabulated for several radial distribution functions, including distributions appropriate for random close packing [Berryman, 1983; Torquato and Stell, 1985]. An approximate solution for small $\mathbf{r}$ and small $\phi$ for hard spheres is

$$
S_{2}(r)=1+\alpha \ln \phi
$$


[Torquato and Stell, 1985], where $\alpha$ is again given by Eq. (5).

Variation of $S_{2}$ with $\mathrm{r}$ is shown in Fig. 1 for both randomly packed impenetrable and fully penetrable spheres with uniform radius, and many of the useful features of this function are illustrated. The lag or separation distance $r$ between two points is given in units of sphere radii. At zero lag, the two-point correlation function reduces to the one-point correlation function and provides a measure of the porosity. For the hard spheres, at large lag the points are uncorrelated, and the function asymptotically. approaches the square of the porosity. The curve for the penetrable spheres will attain this value for any $r$ larger than the grain diameter because for penetrable spheres the pore space at lengths larger than $2 R$ is uncorrelated. For any granular porous material, the overall shape of the two-point correlation function can be related, at least qualitatively, to the particle morphology.

For our idealized fully penetrable sphere example, we can see from Eq. (3) that

$$
S_{2}^{\prime}(\mathrm{r})=\alpha^{\prime}(\mathrm{r}) S_{2}(\mathrm{r}) \ln \phi \text {. }
$$

The derivative in Eq. (7) vanishes for $r \geq 2 R$. At $r=0$, the penetrable sphere model produces the exact result

$$
S_{2}^{\prime}(0)=\frac{3}{4 R} \phi \ln \phi .
$$

$S_{2}$ also can provide estimates of pore diameters. For the penetrable sphere model, Eq. (8) shows that the line tangent to the $S_{2}$ curve at $r=0$ will intersect the horizontal $S_{2}(r)=\phi^{2}$ asymptote at $r_{c}$ where the critical $\operatorname{lag} r_{c}$ is determined by

$$
\begin{gathered}
\phi^{2}=\frac{3}{4 R} \phi r_{c} \ln \phi+\phi \\
\text { or } \\
r_{c}=\frac{\phi-1}{\frac{3}{4 R} \ln \phi}=\frac{\phi(\phi-1)}{S_{2}^{\prime}(0)} .
\end{gathered}
$$

A similar analysis for the hard sphere model will provide the same formula for $r_{c}$ in terms of $S_{2}(0)$. Comparison of Eq. (10) with the definition of $s$ in Eq. (3) shows that the denominator of Eq. (10) is simply -s/4, and therefore

$$
r_{c}=\frac{4 \phi}{s}(1-\phi)
$$

for both the penetrable and impenetrable sphere models. In Fig. 1, the line giving the initial slope of the $S_{2}$ curve is extended to intersect with the horizontal line through the asymptotic value, and the value of the lag $r$ at the point of intersection is $r_{c}$. We define $r_{c}$ as an effective pore diameter because it is related to the hydraulic diameter $D_{H}$, which is defined as

$$
\mathrm{D}_{\mathrm{H}}=\frac{4 \phi}{\mathrm{s}}
$$

[Dullien, 1979]. Comparison of Eqs. (11) and (12) shows that $r_{C}$ is of the same order as $D_{H}$, and $r_{c}$ approaches $D_{H}$ for small $\phi$. Aithough this relationship between $r_{c}$ and $D_{H}$ was derived for idealized sphere models, the parameter $r_{c}$ determined from the correlation function for any material can be used as an estimate of the pore size or hydraulic diameter.

We illustrate how the correlation length is related to grain size by considering the $S_{2}$ curves for the idealized penetrable and impenetrable sphere models. For a material composed of fully penetrable spheres, the lag $r$ at which the $S_{2}$

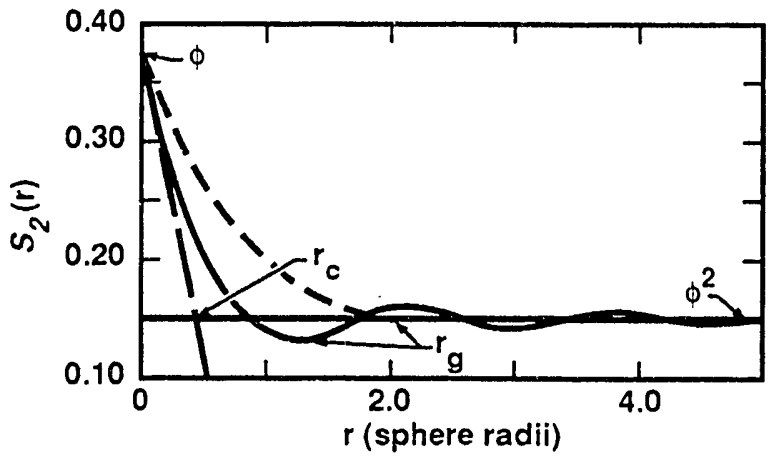

Figure 1. Two-point correlation functions for fully penetrable (short dashes) and impenetrable (solid curve) s, ihere models [after Berryman and Blair, 1986]. Porosity is 0.38 for both curves. Parameter $r_{c}$ gives estimate of mean pore diameter; $r_{g}$ gives estimate of spherical particle size. Slope near origin is shown by long dashed line. 
curve attains a value of $\phi^{2}$ will be designated $r_{g}$ (Fig. 1). For the hard sphere model, the curve has a minimum at a lag that we will also call $\mathbf{r}_{\mathrm{g}}$. For the penetrable sphere model, the mean grain diameter is given by $r_{g}$. For the hard sphere model, however, Fig. 1 shows that the mean grain radius is approximately $\mathrm{r}_{\mathrm{g}} / 1.3$, assuming a radial distribution corresponding to random close packing for the hard spheres. The $\mathrm{S}_{2}$ curve for the hard sphere model also shows oscillatory behavior and other minima at larger values of $r$. Although most materials will be more complicated than these two ideal models having uniform spherical grains, we can use the insight gained from these models to analyze other $S_{2}$ curves to estimate particle sizes and characteristics. If the curve for a more general material shows a distinct minimum dropping below the asymptotic $\phi^{2}$ value, this strong negative correlation implies that the particles are approximately uniform in size, and the lag at which the minimum occurs gives an estimate of the mean particle size. The $S_{2}$ curve for a well-sorted sandstone might show such a minimum. If the curve shows oscillatory behavior similar to the curve for the hard sphere model, this indicates that the particles are rounded rather than angular. The $S_{2}$ curve for a poorly sorted material will not have a distinct minimum, but will instead gradually (and monotonically) approach the $\phi^{2}$ asymptote much more slowly than the curve for the idealized penetrable sphere model approaches the asymptote. The value of $r$ as the curve approaches the asymptote gives an estimate of the maximum particle diameter, but this value will not be sharply defined as it is for the idealized penetrable sphere model. In summary, we see that $S_{2}$ can provide quantitative information regarding the pore and grain sizes and the specific surface, as well as an indication of the sorting and angularity of particles in the sample being analyzed.

\section{Kozeny-Carman relations}

The permeability of a porous material is defined as the constant of proportionality relating the pressure gradient to the rate at which a fluid of known viscosity will flow through the material [Darcy, 1856; Dullien, 1979]. The most successful methods for analyzing permeability make use of various empirical relations between fluid permeability and other physical properties of porous materials. Such empirical relationships, generally termed Kozeny-Carman relations [Carman, 1956; Dullien, 1979], are derived from simple models of pore structures. Kozeny-Carman relations have the general form

$$
k=\frac{\phi r_{H}^{2}}{c}
$$

where $k$ is the permeability, $\phi$ is the pornsity, $r_{H}$ is the hydraulic radius, and $\mathrm{c}$ is a constant related to pore geometry.

Paterson [1983] and Walsh and Brace [1984] modeled pores as straight cylindrical tubes having smooth walls in order to obtain a formula relating permeability $k$ to porosity $\phi$, formation factor $F$, and specific surface area s:

$$
\mathrm{k}=\frac{\phi^{2}}{\mathrm{cFs}} \text {. }
$$

Here, the factor $c$ depends on the shape of the tube cross section. Typically, $c=2$ for circular tubes and $c=3$ for flat cracks. As mentioned above, the slope of $S_{2}$ near the origin is proportional to the specific surface area of the material. Thus, $S_{2}$ can be used to provide this parameter for Eq. (14), when estimating permeability. However, care must be used in application of this result, and it is easy to see that imaging techniques such as scanning electron microscopy do not resolve features of the material that contribute to the intrinsic specific surface area measured using such means as a gas adsorption method (e.g., BET). Moreover, images produced at a resolution appropriate for determination of porosity and grain and pore dimensions need to sample many grains $(\sim 100)$, while features that contribute to the molecularspecific surface are not resolved at comparable magnifications. Despite these difficulties, useful information regarding the pertinent degree of roughness of the pore surface can be derived from images produced using standard techniques. Berryman and Blair [1987] have shown that for the Kozeny-Carman model for fluid flow in porous media, it is not the absolute specific surface area but the roughness of the pore walls relative to the mean pore size that influences the permeability. This can be seen by considering Fig. 2. Berryman and Blair [1987]

have shown that permeability in a tube of radius $R_{i}$ is less than that for a tube of radius $R_{o}$ 


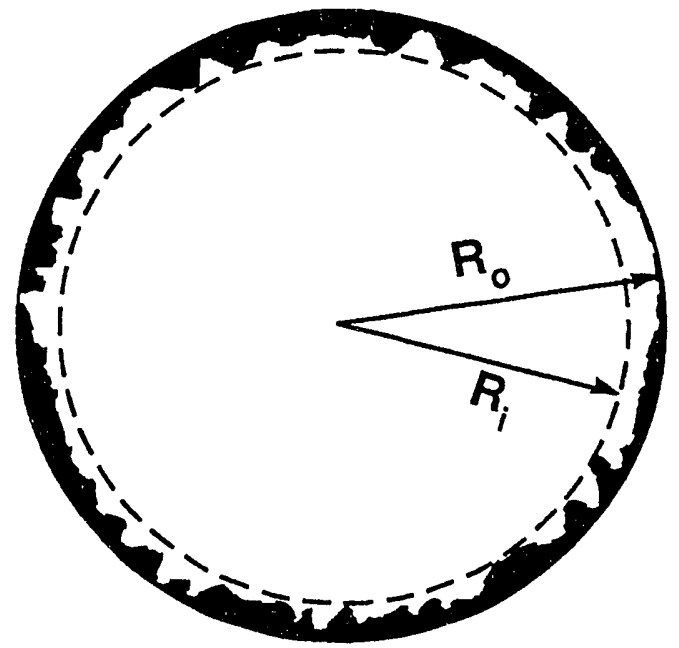

Figure 2. Cross section through a rough-walled tube with radius bounded by circles of radii $\mathbf{R}_{\mathbf{i}}<\mathbf{R}_{\mathrm{o}}$ [after Berryman and Blair, 1987].

if $\mathrm{R}_{\mathrm{i}}<\mathrm{R}_{\mathrm{o}}$. They define an image specific-surface area that is appropriate for conduit flow and show that this specific surface area can be measured from images of cross sections if the images have a pixel size $h$ such that $h=r_{c} / 200$, where $r_{c}$, defined in Eqs. (9-11), is measured on an image that contains $\sim 100$ grains. We will use this image specific-surface area for $s$ in Eqs. (3) and (14).

In addition to an estimate of the specific surface area, Eq. (14) requires an estimate of $F$. The formation factor $F$, defined as the ratio of the electrical conductivity of a conducting pore fluid to the effective electrical conductivity of an insulating material saturated with the fluid, gives a measure of the tortuosity of the connected pore space. If measured values are unavailable, $F$ can itself be estimated using a self-consistent model in which each grain of the porous material is modeled as an insulator coated with a thin conducting film consisting of smaller insulators suspended in a conducting fluid [Sen et al., 1981]. This model gives

$$
F=\phi^{-m},
$$

where $m=1.5$ for uniform spherical grains [Sen et al., 1981]. Such a power law was found empirically by Archie [1942], who used a cementation factor $m$ between 1.8 and 2 for consolidated sandstones. Typically for rocks, values of $m$ near 2 yield good estimates of $F$ [Brace, 1977; Wong et äl., 1984].

Given the capabilities of commonly available systems for imaging rock cross sections, we find that when using $S_{2}$ to estimate permeability and other rock properties, images should be used at two magnifications: a lowmagnification image that samples $\sim 100$ grains to determine porosity, effective grain size, and effective pore diameter; and a higher-power image to determine image specific-surface area. The appropriate choice of magnification for the high-power image is related to the effective pore diameter estimated from the low-power image. These constraints arise because many imaging systems handle images that are fixed in size (e.g., $512 \times 512$ pixels). In this study, we used images showing 100 grains as the lowpower images, and our high-power images showed $\sim 1-3$ grains.

\section{Experimental Methods}

\section{Synthetic sandstones}

We first evaluated the spatial correlation function method by applying it to study a suite of synthetic sandstone samples. These samples were made by sintering glass beads at peak temperatures of $700-760^{\circ} \mathrm{C}$ to achieve various porosities and cooling slowly through the annealing temperature of about $550^{\circ} \mathrm{C}$ to reduce microcrack development. The beads were placed in Pyrex beakers and surrounded by fire bricks to minimize thermal gradients in the furnace during sintering. Cataphote, Inc., of Jackson, Mississippi, manufactured the beads from sodalime plate glass. According to the manufacturer's specifications, the beads had diameters of $230 \pm 20 \mu \mathrm{m}$ and were nearly spherical, as the diameters of individual beads deviated $<5 \%$ from a uniform spherical shape. The glass density was $2480 \pm 20 \mathrm{~kg} / \mathrm{m}^{3}$. Right circular cylinders with diameters of $2.54 \mathrm{~cm}$ were cored from the sintered beads for use in elastic property studies [Berge et al., 1993]. Thin sections of horizontal and vertical cross sections were made from core ends. Masses and volumes of the cylindrical samples were determined with an uncertainty of $<0.1 \%$ and used with the glass density to compute porosities with uncertainties of $0.6-1 \%$ (Table 1). 
These observed porosities $\phi_{\text {obs }}$ of the cylindrical samples are assumed to be representative of the thin sections made from the core ends.

Permeabilities of the four cylindrical samples were measured using a permeameter with filtered, deionized water as the pore fluid.

\section{Preparation and imaging of thin sections}

We subsequently analyzed images of both synthetic and natural sandstones. Images used in the analysis were produced by first impregnating the samples with low-viscosity epoxy and then preparing standard petrographic thin sections. These sections were polished with $1 / 4-\mu \mathrm{m}$ diamond grit and left uncovered. The sections were then examined using a scanning electron microscope, which was operated in the backscatter mode to produce high-contrast images of the cross-sectional planes. Typical raw images of glass-bead and sandstone samples are shown in Fig. 3(a and d). The grains appear bright and the pore space appears dark in these images, because the grains reflect the electrons while the epoxy in the pore space absorbs them. The SEM images were printed on photographic paper and then digitized using a scanning digitizer. Once in digital form, the images were cropped and a threshold filter was applied to produce binary images that were used in the analysis. Figure 3 compares raw and filtered binary images for a glass-bead sample and a sandstone. In general, the synthetic sandstone samples required less image processing than the natural sandstones in order to produce useful binary images for computing correlation functions. This is due in part to the fact that the synthetic sandstone thin sections contain only glass and epoxy, while the natural materials contain multiple constituents. Examples of binary images are shown in Fig. 3(b and e). The histograms of pixel intensities shown in Fig. 3( $c$ and $f$ ) give an indication of how raw images were filtered to produce binary images. $S_{2}$ was computed using the algorithm described by Berryman [1985b]. Estimates of $r_{c}$ and $r_{g}$ were determined for each low-power image. To determine the initial slope, a linear fit was made to the first few points of $S_{2}$ computed for the highmagnification images. The image specific surface was determined using Eq. (3). Permeability was then calculated using Eq. (14), and $D_{H}$ was determined using Eq. (12).

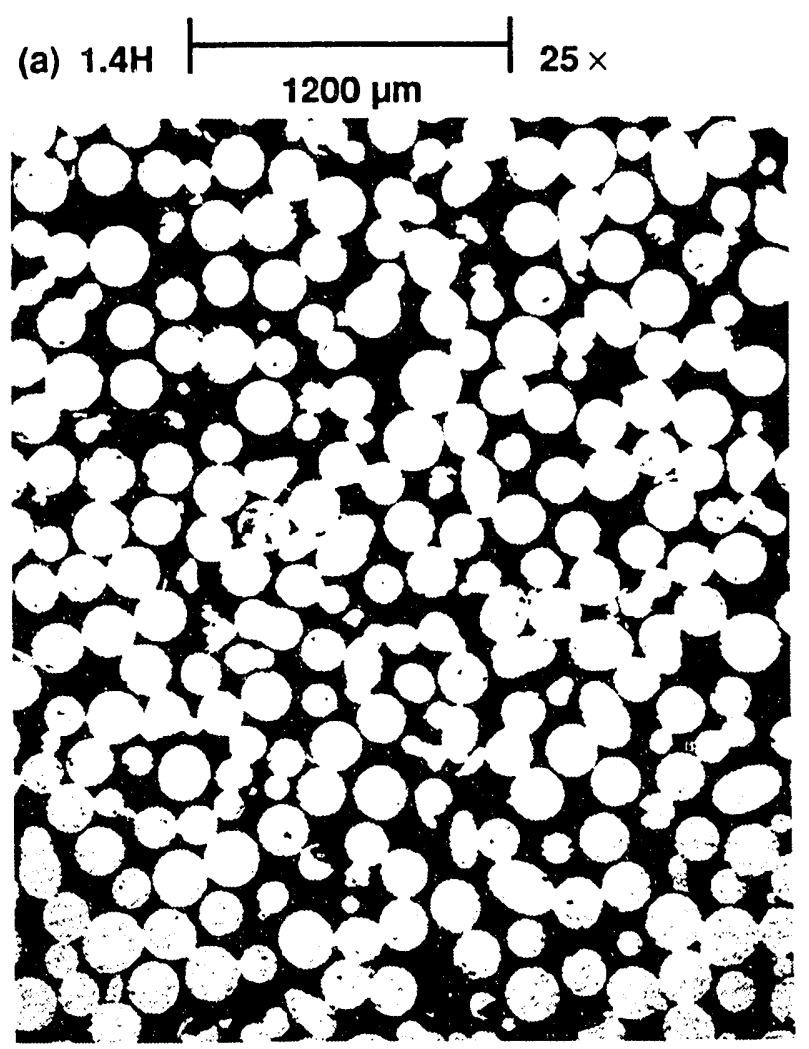

(b)

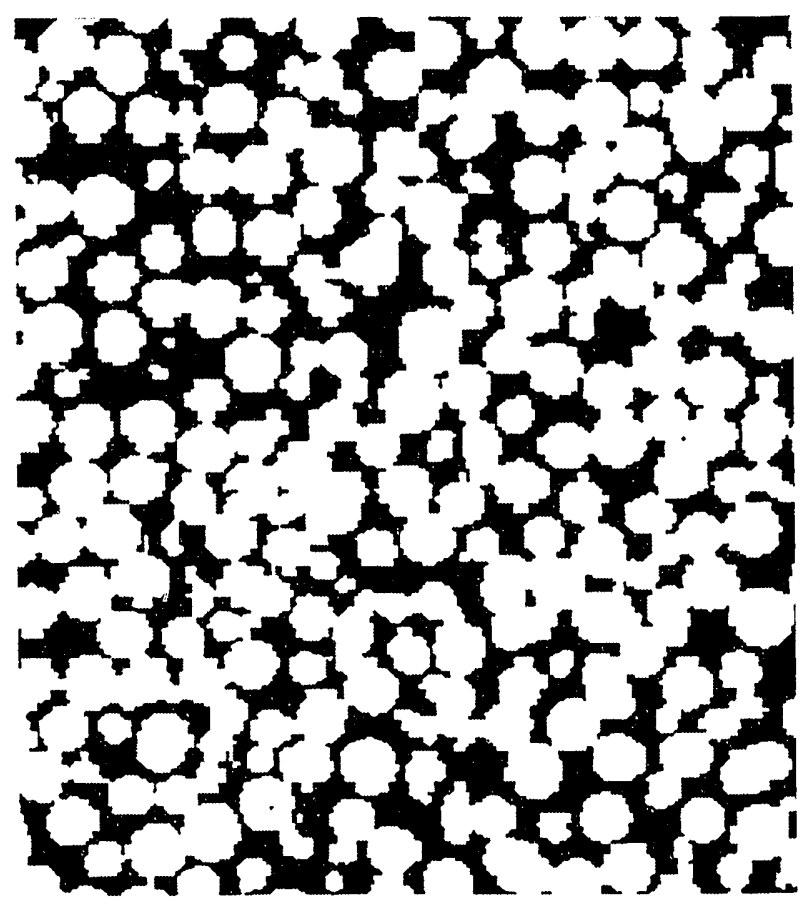

Figure 3. Comparison of raw and binary images of sandstones. (a) Raw $25 \times$ magnification SEM image of sintered glass-bead sample 1.4 and (b) corresponding filtered binary image. 
(c)

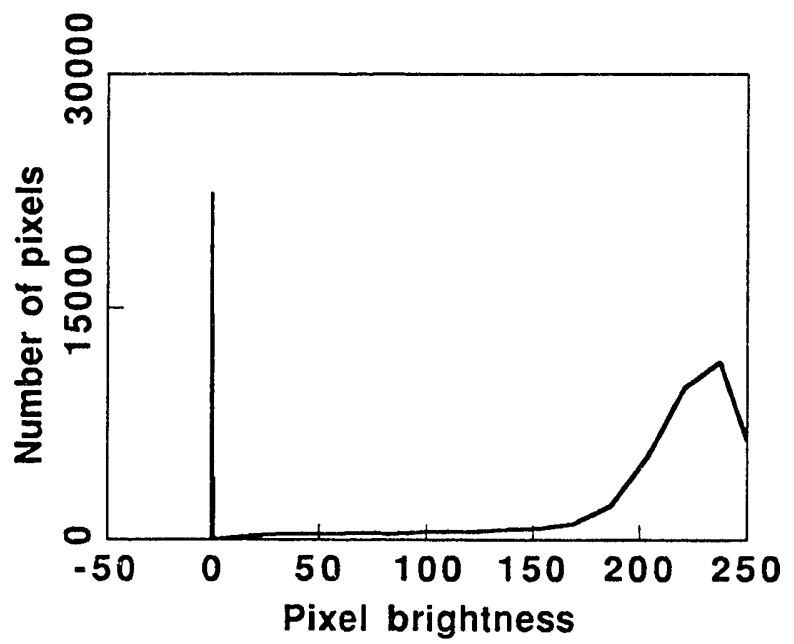

(d) Raw image BF1

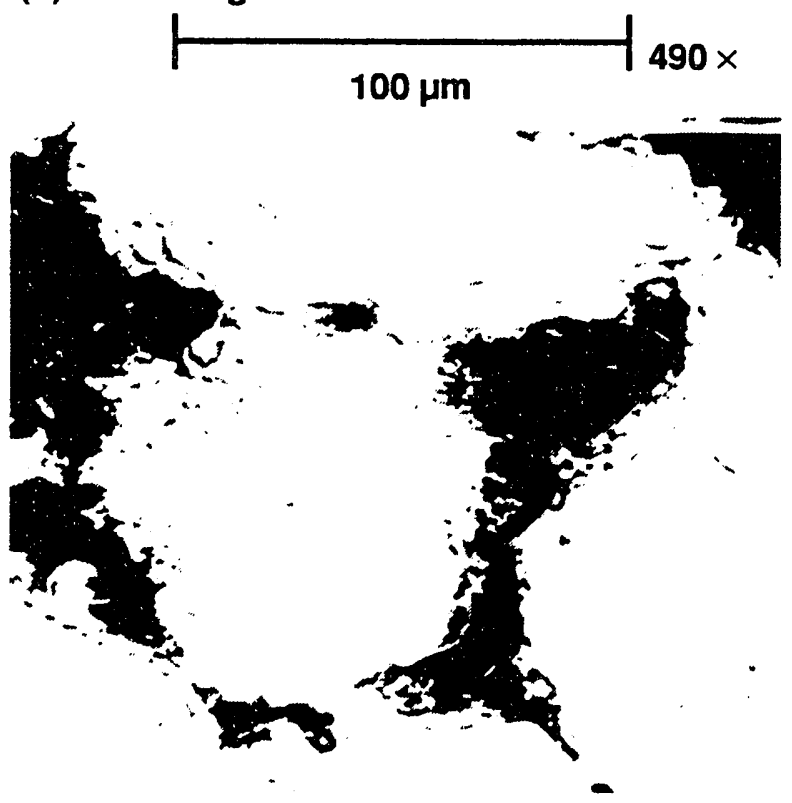

(e) Binary image BF1
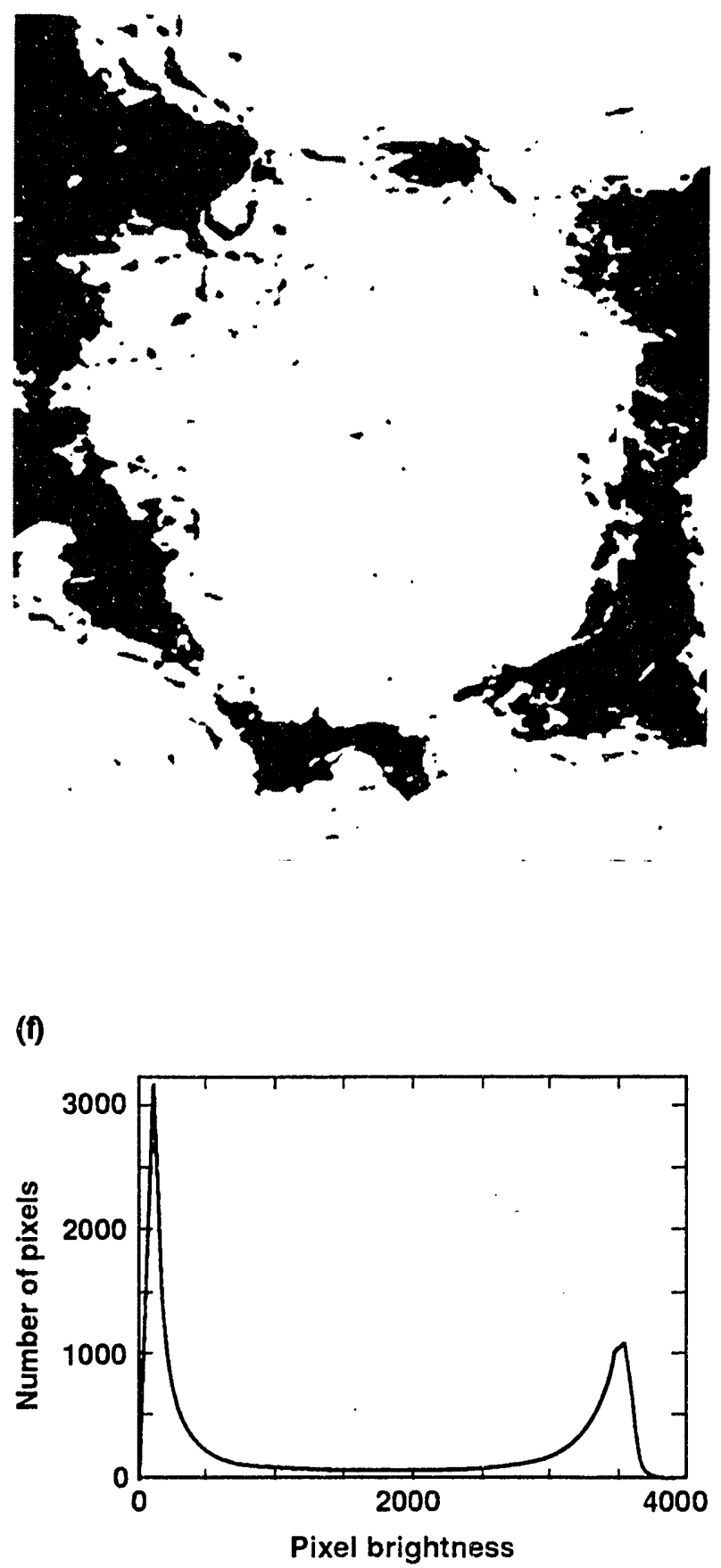

Figure 3, continued. (a) and (b) are compared with (c) histogram of pixel brightness for digitized raw image prior to filtering. (d) Raw $490 \times$ magnification SEM image of Berea sandstone sample BF1 with (e) corresponding filtered binary image and (f) histogram [after Berryman and Blair, 1986]. 


\section{Results}

As discussed above, two-point correlation functions can be used to determine several parameters that describe the microstructure of a porous material. Furthermore, the porosity and specific surface area, together with the formation factor, provide the information required by a simple Kozeny-Carman flow model to estimate intrinsic permeability. We have computed $S_{2}$ for cross sections of several synthetic and natural sandstones to assess the effectiveness of this method for characterizing rock and rock-like synthetics. We computed the two-point correlation functions for images of four synthetic and six natural sandstones. Parameters describing the microstructure were determined, as well as porosities and image specific-surface areas. First we will present the results of microstructural analysis for the synthetic sandstones followed by results for natural sandstones, and then we will discuss the permeability estimates.

\section{Microstructure of Synthetic Sandstones}

One 0 : the goals of the synthetic sandstone analysis was to assess the isotropy and homogeneity of the samples. We were concerned about thermal stratification and about the presence of microcracks formed during the heating/cooling cycle. Thus, horizontal and vertical thin sections were produced for each sample. For each of the horizontal and vertical thin sections, five SEM images were recorded at low magnification and five at high magnification. Each of the four synthetic sandstones is represented by 20 SEM images: 5 horizontal cross sections at $25 \times$ magnification, 5 horizontal cross sections at $300 \times$ magnification, 5 vertical cross sections at $25 \times$ magnification, and 5 vertical cross sections at $300 \times$ magnification. Comparison of the correlation functions measured using different images provided a means to assess both the homogeneity and the isotropy of the samples.

The $S_{2}$ curves indicate that the samples are practically isotropic and homogeneous. Figure 4 compares the correlation functions determined from low-magnification images of horizontal and vertical cross sections for sample 1.4. [Images of the horizontal cross section for this sample are shown in Fig. 3(a and b). At this porosity, the sample approximates random close packing of hard spheres.] The two curves in Fig. 4 are nearly identical, indicating that the sample did not have significant thermal stratification caused by thermal gradients in the furnace. This result is not surprising since the sample volume, $\sim 13 \mathrm{~cm}^{3}$, is very small compared to the furnace volume, $\sim 130,000 \mathrm{~cm}^{3}$. We also can see that these curves behave very much like the curve for the hard sphere model shown in Fig. 1.

We also wanted to gain insight into the behavior of the correlation function for a sequence of real materials ranging from those approximating the hard sphere model to those approximating the penetrable sphere model. In Fig. 5( $a$ and $b)$, low-magnification SEM images of two synthetic sandstones show how the microstructure differs for different porosities. We found that the microgeometry of the lowest porosity sample is similar to the fully penetrable sphere model, while the microgeometries of the three higher porosity samples are more like the impenetrable sphere model. Figure $5(\mathrm{c})$ contrasts the correlation functions for low magnification images of samples 1.1 and B. In low-porosity sample 1.1, the beads are fused tightly together and no longer retain their original nearly spherical

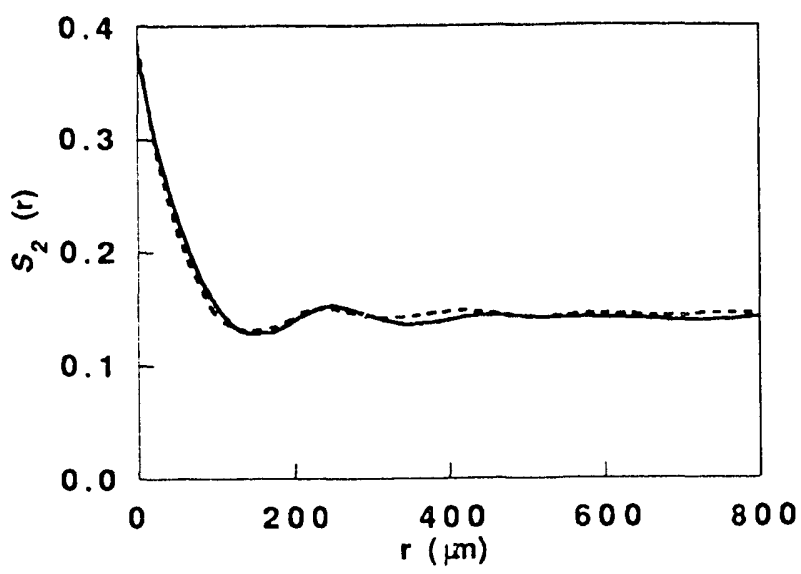

Figure 4. Sample isotropy demonstrated using correlation functions computed for $25 x$ magnification images of a horizontal (dashed curve) and a vertical (solid curve) cross section for synthetic sandstone sample 1.4. 
(a) $\mathrm{BH}$
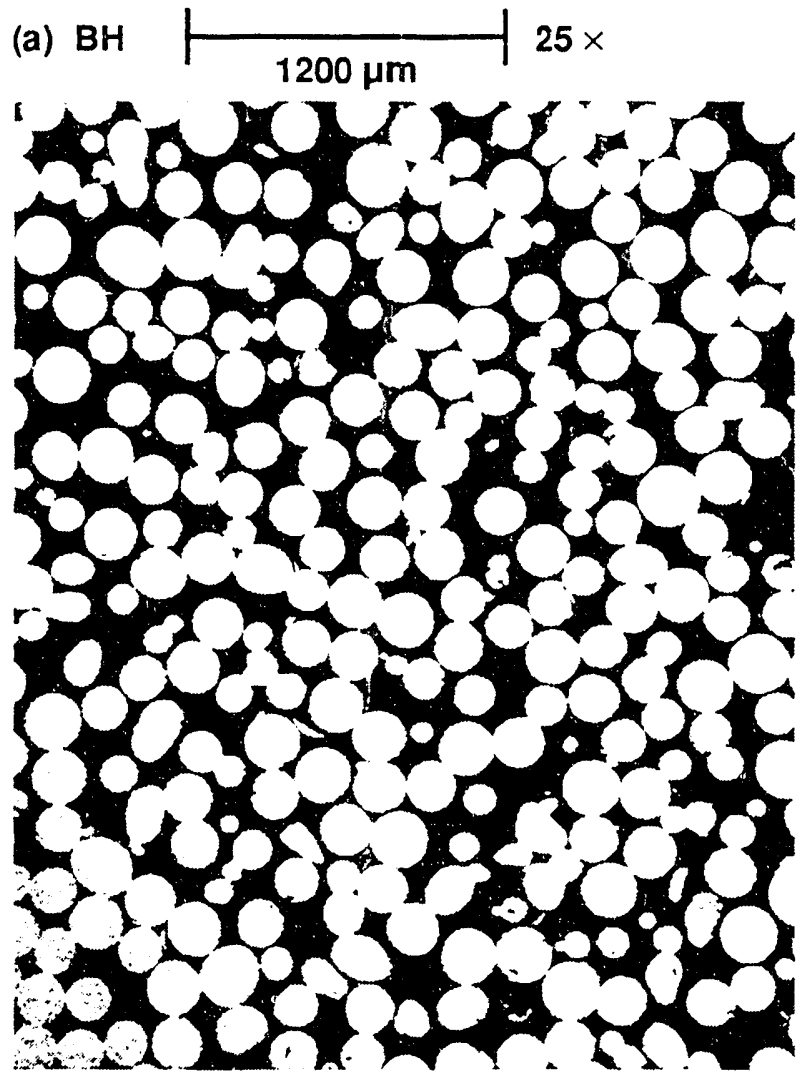

(b) $1.1 \mathrm{H}$

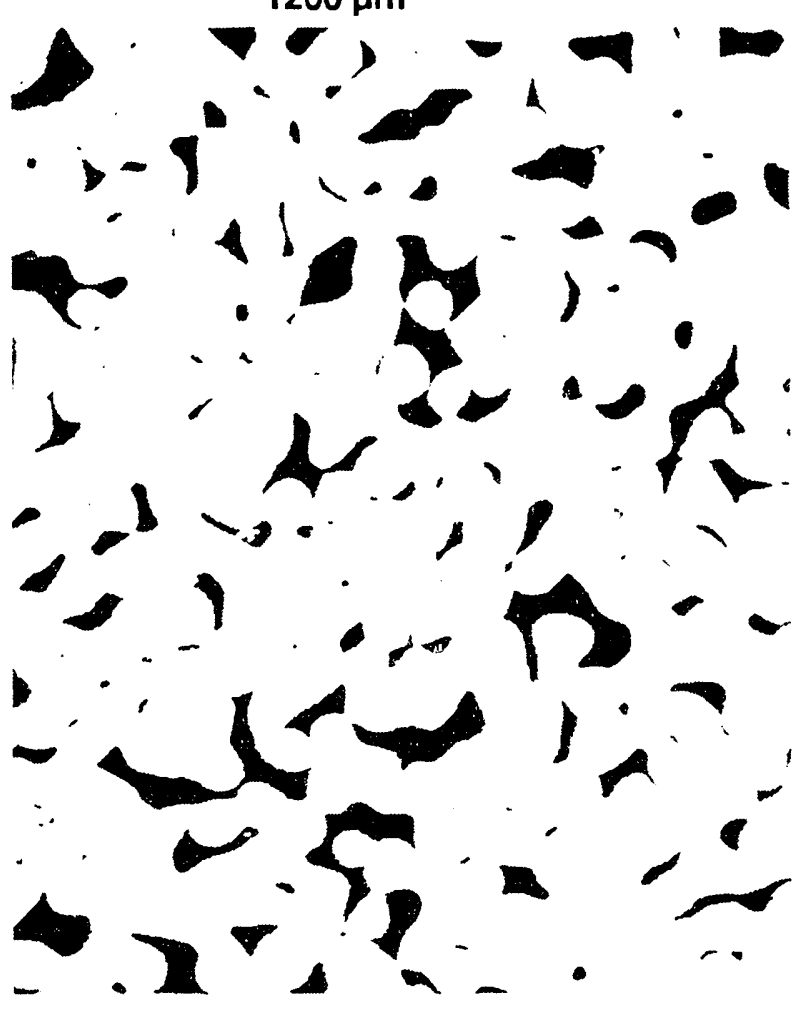

(c)

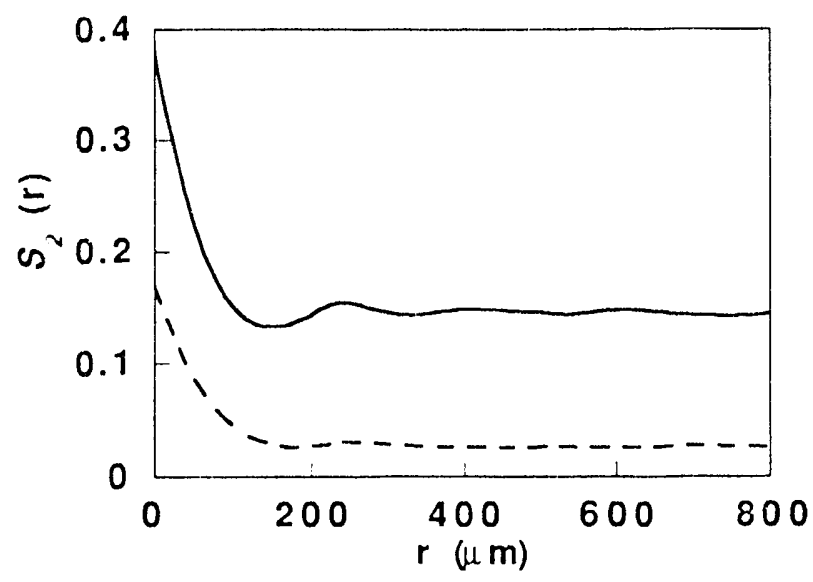

Figure 5. Synthetic sandstone samples representing the impenetrable and penetrable sphere models. $25 \times$ magnification SEM images of (a) sample B and (b) sample 1.1 together with (c) correlation function curves for samples B (solid curve) and 1.1 (dashed curve).

shapes. The correlation function for this sample attains the asymptotic $\phi^{2}$ value for $r$ near $200 \mu \mathrm{m}$ and larger and behaves like the fully penetrable sphere curve shown in Fig. 1. But high-porosity sample B is barely consolidated, and the grains are still approxirnately spherical. The correlation function for sample $B$ has a minimum that falls below the asymptotic value and behaves more like the impenetiable sphere curve in Fig. 1.

We used the low-magnification images to find porosity estimates $\phi_{\text {calc }}$ and effective pore and grain diameters for the four synthetic sandstones. Figures 6 and 7 show correlation functions for samples B and 1.1, respectively (corresponding SEM images are displayed in Fig. 5). Results for all four synthetic sandstone samples are presented in Table 1, along with the observed porosities $\phi_{\text {obs }}$ determined from the masses and volumes of the cylindrical samples. (These four samples, described in detail by Berge et al. [1993], are a representative subset of a suite of 24 synthetic sandstone samples. The sample labels used here are related to the labeling scheme used for the entire suite of samples.)

The ranges of values given in Table 1 show to what extent SEM images of each sample vary. The porosities were found from the values of the correlation functions at the origin; the 


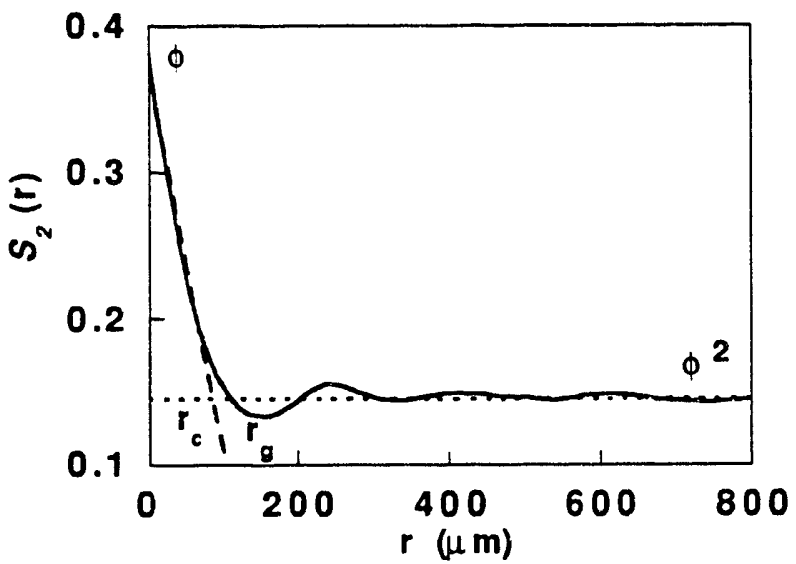

Figure 6. Correlation function and grain parame ${ }^{a} ? r_{i}$ for synthetic sandstone sample $B$. Pore diameter estimate shown by $r_{c} ; r_{g}$ gives grain size estimate.

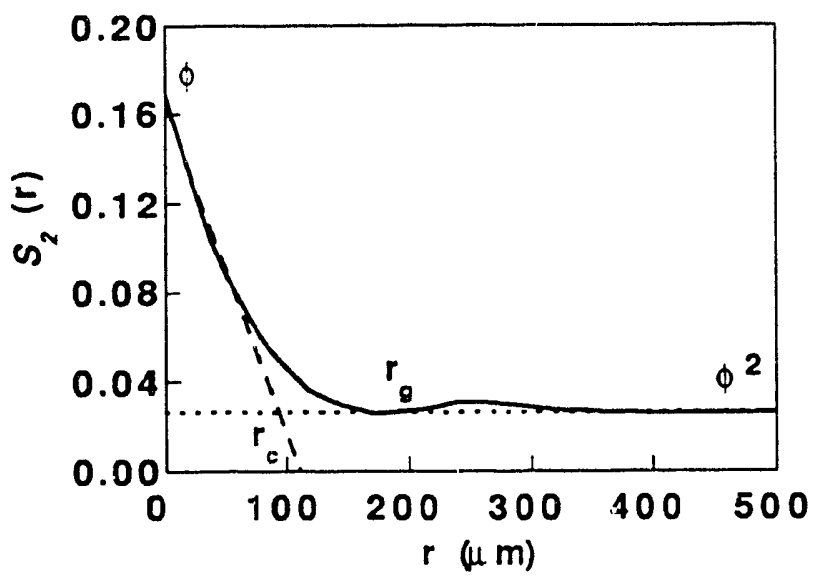

Figure 7 . Correlation function and grain parameters for synthetic sandstone sample 1.1. Pore diameter estimate is shown by $r_{c} ; r_{g}$ gives grain size estimate. correlation function values approach $\phi^{2}$ for large distances from the origin. Table 1 lists the minima of the curves at $r_{g}$. For the synthetic sandstones, the effective grain diameters were found by assuming that the more porous samples are good approximations of the hard sphere model, whereas the densest sample emulates the penetrable sphere model. For these ideal models, the sphere radius is determined by dividing ${ }_{g}$ by 1.3 or by 2 , for the hard or the penetrable sphere models, respectively. Effective pore diameters were found for all samples from the points at $r_{c}$, where lines tangent to the $S_{2}$ curves at the origin intersect the horizontal asymptotes. The porosities estimated using the correlation functions match the observed porosities in Table 1 to within a few percent for all samples. The beads in the high-porosity samples retained their original nearly spherical shapes and original diameters of $230 \pm 20 \mu \mathrm{m}$ when they were sintered. The estima ${ }$. If $2 r_{g} / 1.3$ in Table 1 predicts the grain diameters for these samples, indicating that they behave like the impenetrable sphere model. If we assume that the lowest-porosity sample approximates the fully penetrable sphere model, then the grain diameter found using the $r_{g}$ value is slightly smaller than the original bead diameter. The lowest-porosity sample differs from the ideal penetrable sphere model because the beads fuse together and deform during sintering instead of behaving like the idealized interpeneirating spheres.

As discussed previously, we used highermagnification images for determining the image specific-surface areas. Figures 8-11 show the high-magnification SEM images of the synthetic sandstone samples and the two-point

Table 1. Pore and grain parameters for synthetic sandstones made from sintering $230 \pm 20-\mu \mathrm{m}$ glass beads.

\begin{tabular}{ccllccccc}
\hline Sample & \multicolumn{1}{c}{$\phi_{\text {obs }}$} & $\phi_{\text {calc }}$ & $(\mu \mathrm{m})$ & $\begin{array}{c}\mathrm{r}_{\mathrm{g}} \\
(\mu \mathrm{m})\end{array}$ & $\begin{array}{c}\text { Grain Diam.* } \\
(\mu \mathrm{m})\end{array}$ & $\begin{array}{c}\mathrm{s} \\
\left(\mu \mathrm{m}^{-1}\right)\end{array}$ & $\begin{array}{c}\mathrm{D}_{\mathrm{H}} \\
(\mu \mathrm{m})\end{array}$ \\
\hline B & $0.39 \pm 0.01$ & 0.37 & 80 & $145-158$ & $223-243$ & $0.0253 \pm 0.004$ & $60 \pm 10$ \\
1.4 & $0.365 \pm 0.006$ & $0.35-0.39$ & $60-80$ & $145-155$ & $223-238$ & $0.0205 \pm 0.004$ & $71 \pm 14$ \\
1.2 & $0.285 \pm 0.006$ & 0.33 & $70-80$ & $132-158$ & $203-243$ & $0.0168 \pm 0.008$ & $79 \pm 4$ \\
1.1 & $0.173 \pm 0.007$ & 0.17 & 92 & $170-185$ & $170-185$ & $0.0162 \pm 0.004$ & $43 \pm 10$ \\
\hline
\end{tabular}

*Calculated using $2 \mathrm{r}_{\mathrm{g}}$ 11.3 for samples $\mathrm{B}, 1.4,1.2 ; \mathrm{r}_{\mathrm{g}}$ for sample 1.1 . 
(a) $\mathrm{BH}$
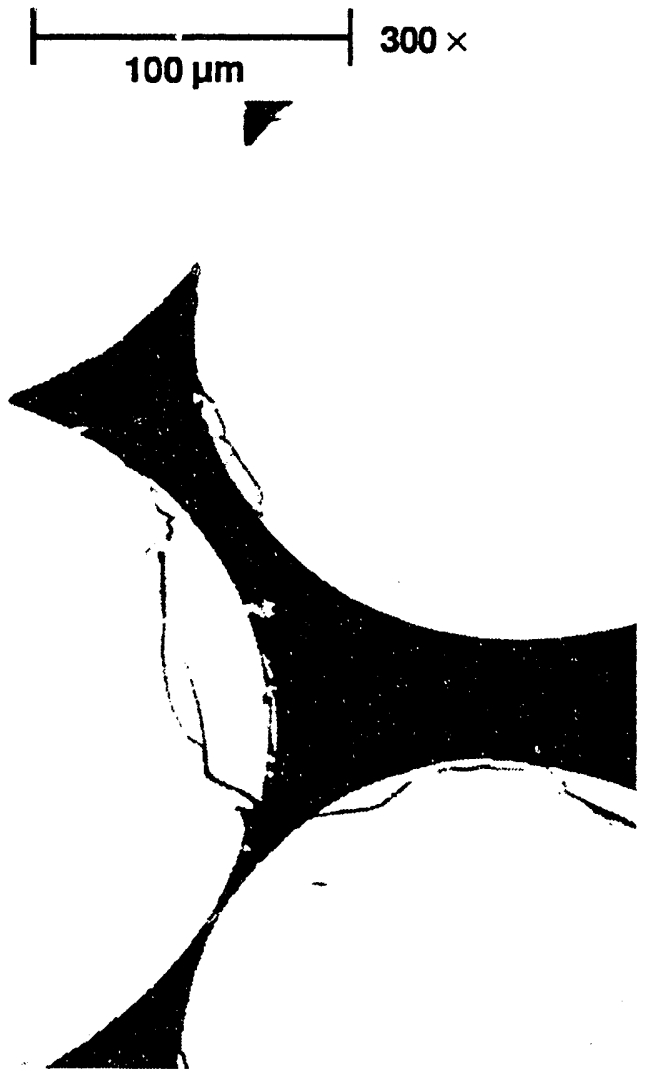

(b)

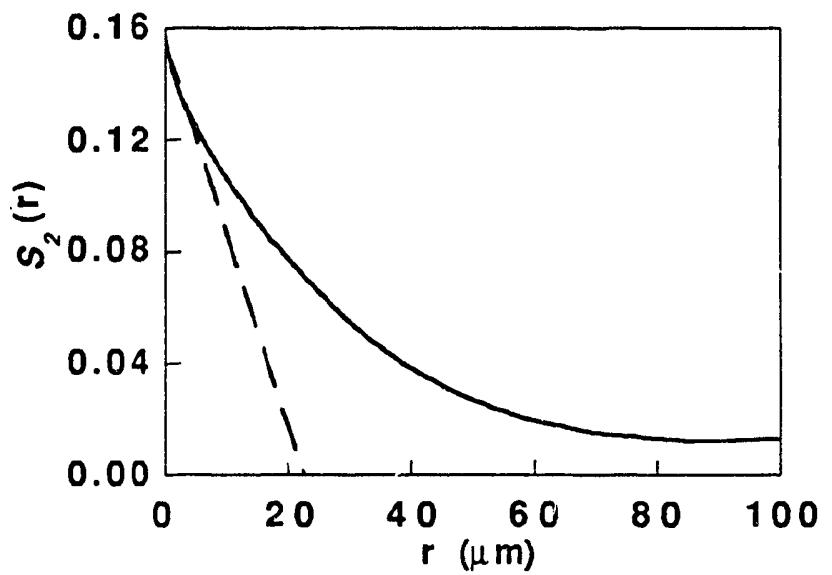

Figure 8. Finding specific surface for synthetic sandstone sample $B$. (a) $300 \times$ magnification SEM image and (b) related correlation function (solid curve) for sample $B$, with slope near the origin shown by a dashed line. (a) $1.4 \mathrm{~V} \mid$\begin{tabular}{l|l}
$100 \mu \mathrm{m}$ & $300 x$
\end{tabular}
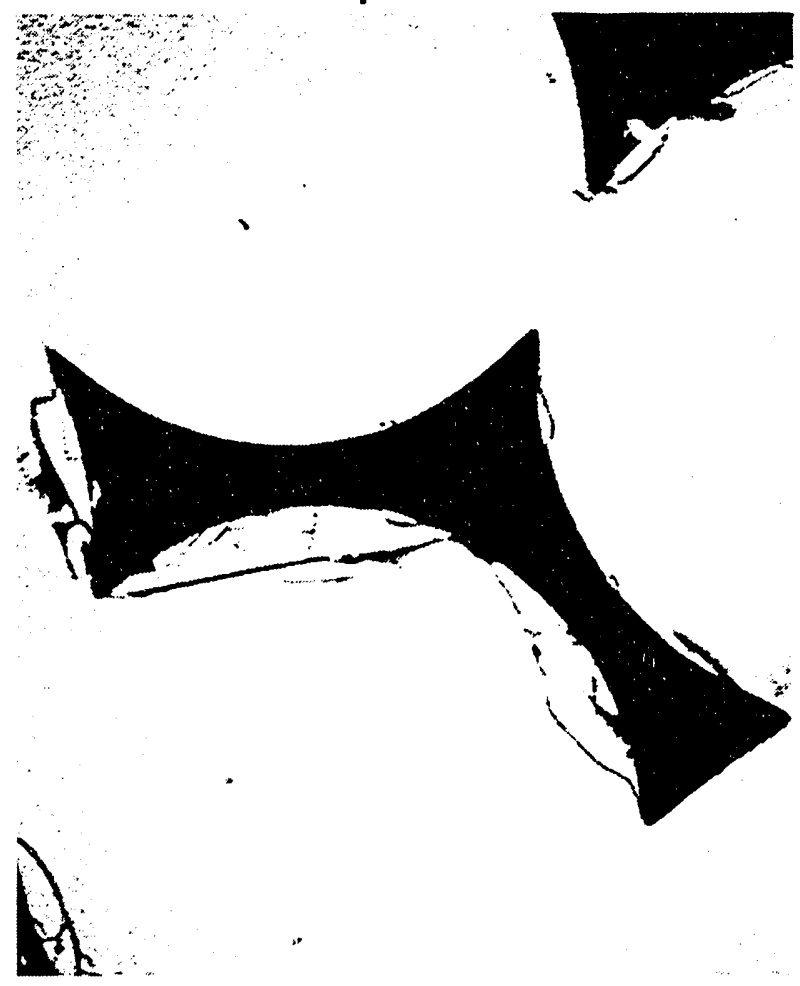

(b)

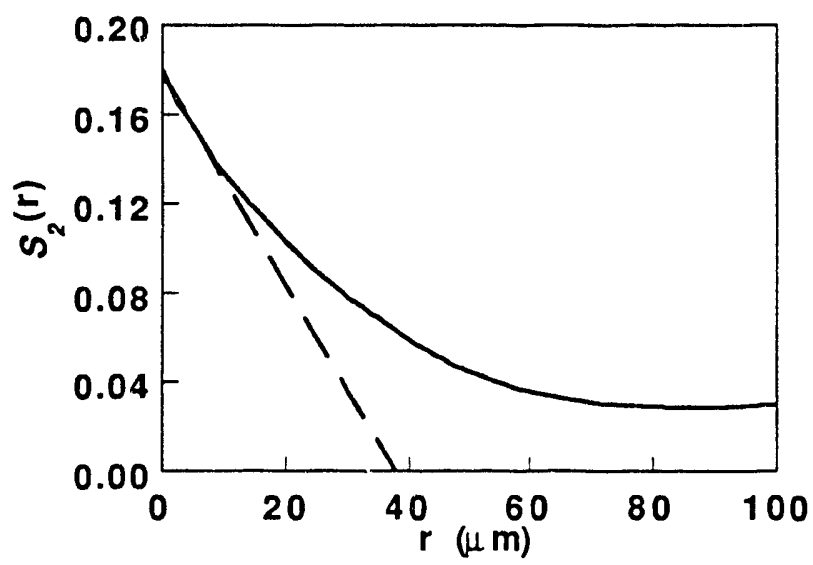

Figure 9. Finding specific surface for synthetic sandstone sample 1.4. (a) $300 \times$ magnification SEM image and (b) related correlation function (solid curve) for sample 1.4, with siope near the origin shown by a dashed line. 

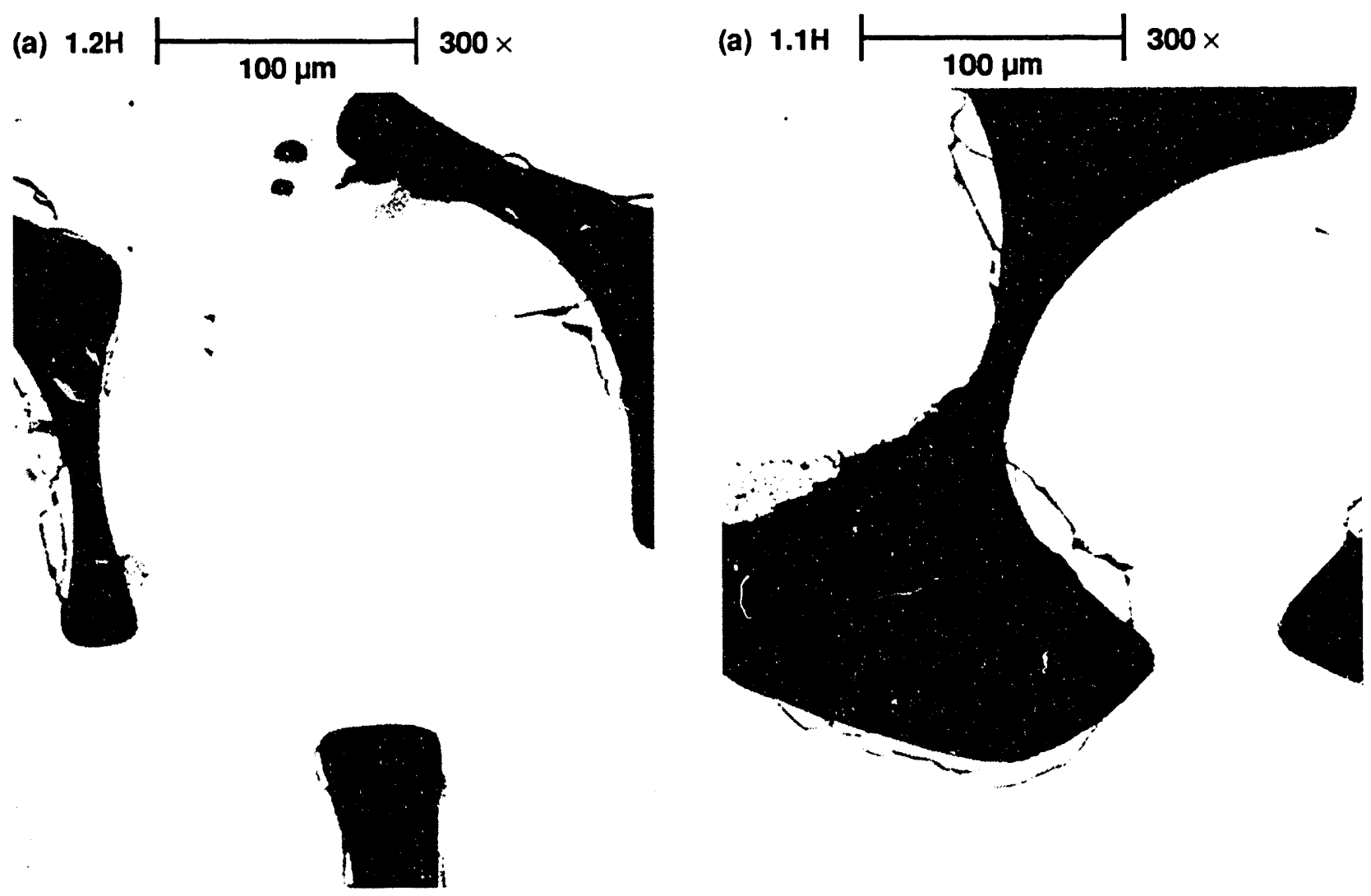

(b)

(b)

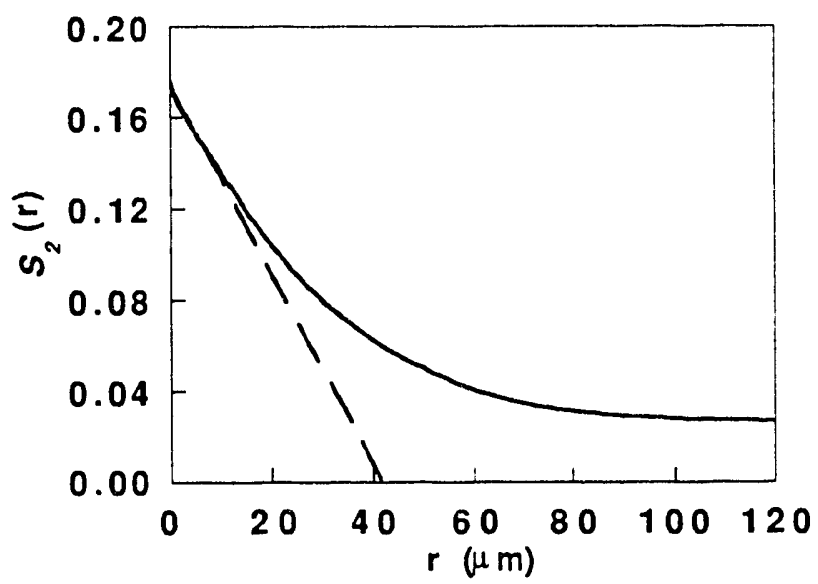

Figure 10. Finding specific surface for synthetic sandstone sample 1.2. (a) $300 \times$ magnification SEM image and (b) related correlation function (solid curve) for sample 1.2, with slope near the origin shown by a dashed line.

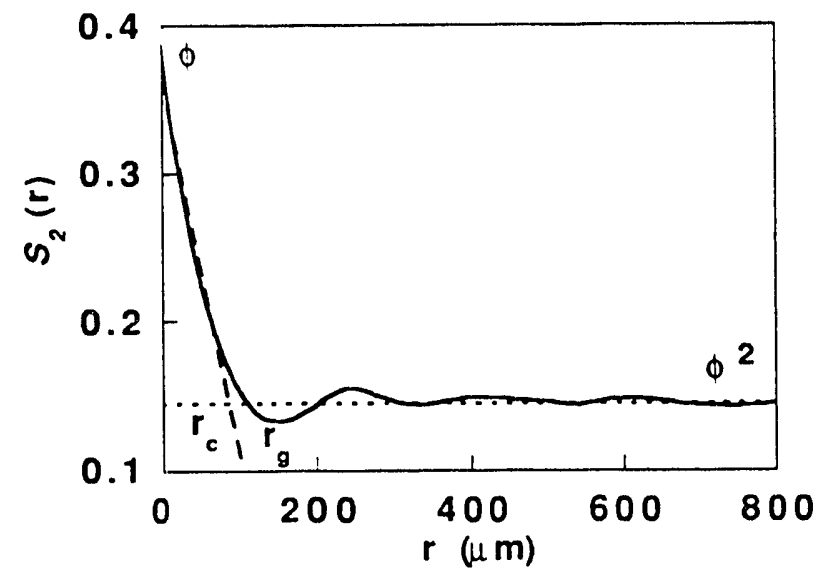

Figure 11. Finding specific surface for synthetic sandstone sample 1.1. (a) $300 \times$ magnification SEM image and (b) related correlation function (solid curve) for sample 1.1, with slope near the origin shown by a dashed line. 
correlation functions calculated from these images. Initial slopes of the correlation functions are indicated by dashed lines. Corresponding values of image specific-surface areas calculated from these slopes using Eq. (3) are presented in Table 1. (Note that the value of $S_{2}(0)$ determined using a high-magnification image is not an accurate measure of the porosity due to poor statistical sampling of the total pore space.) The uncertainties in image specificsurface area values found in Table 1 show how the correlation functions varied for different high-magnification images of each sample. The four synthetic sandstone samples all had very similar image specific-surface area values. This result was expected, as the samples all were constructed from similar glass beads, and the sintering process should not cause variations in the smoothness of the beads for different samples having different porosities.

Sandstones, however, are expected to have much higher specific surface area values, since the grains in natural materials are generally much rougher than glass beads. Values of the hydraulic diameter calculated using Eq. (12) are also included in Table 1. Because the image specific surface did not vary greatly for these samples, the hydraulic diameter was controlled mainly by the porosity, and synthetic sandstone samples having similar porosities had similar hydraulic diameter values.

\section{Microstructure of Natural Sandstones}

\section{Sample descriptions}

We studied samples from six sandstone formations. These were mainly well-sorted, clean quartz arenites, but we included other more complicated samples to evaluate how our correlation function method performed for different kinds of natural materials. The porosities of the natural sandstones ranged from 30 to $9 \%$; we discuss the samples in order of decreasing porosity.

The highest-porosity natural material was the Ironton-Galesville sandstone from the Franconia-Ironton-Galesville confined aquifer in St. Paul, Minnesota. The top of this 63-m-thick aquifer is at a depth of approximately $180 \mathrm{~m}$. The aquifer has an artesian head of $125 \mathrm{~m}$ and consists of horizontal, evenly stratified, fine- to coarse-grained, friable-to-compact sandstone with silty, clayey, and glauconitic zones and weak dolomitic cementation with numerous shale interbeds [Walton, 1981]. Our samples were from depths of $234 \mathrm{~m}(775 \mathrm{ft})$ and $238 \mathrm{~m}$ $(785 \mathrm{ft})$. Permeabilities of these samples were measured by Blair et al. [1985]. These were our most complicated samples.

We also examined two samples of Berea sandstone obtained from Daily and Lin [1985] and from Agrawal et al. [1991]. The Berea sandstone of Ohio is a clean quartz arenite containing about $95 \%$ quartz and about $1 \%$ clay [Caruso et $i . l ., 1985]$. It has moderately wellsorted subangular grains with a mean grain diameter of about 100-120 $\mu \mathrm{m}$ [Coyner, 1984; Caruso et al., 1985]. Estimates of porosity range from abcuit 15-19\%, depending on clay content [Coyner, 1984; Caruso et al., 1985; Daily and Lin, 1985; Wissler, 1987]. Permeabilities and formation factors have been measured by Dullien [1979], Daily and Lin [1985], and Agrawal et al. [1991].

We had one sample of the Frontier sandstone described by Caruso et al. [1985], which contains about $95 \%$ quartz and about $5 \%$ clay. The Frontier sandstone of Wyoming is a clean quartz arenite with subangular to subrounded grains. The grains are well sorted and have a mean diameter of about $130 \mu \mathrm{m}$ [Caruso et al., 1985]. The porosity is about $21.7 \%$. Wissler [1987] measured the permeability and formation factor for our sample.

The Tensleep formation of Wyoming is also a clean quartz arenite, containing $95-97 \%$ quartz and about $2 \%$ clay [Caruso et al., 1985]. Grains have a mean diameter of $115-170 \mu \mathrm{m}$, are well sorted, and subangular to subrounded [Caruso et al., 1985]. Our sample had a porosity of $18.1 \%$ [Caruso et al., 1985], and the formation factor and permeability were measured by Wissler [1987].

In addition, we had a sample of Navajo sandstone. This is a fine-grained, well-sorted clean quartz arenite with quartz cement, containing no clay [Wissler, 1987; Burns et al., 1990]. It has a porosity of about $11.8 \%$ and a grain size of about $150 \mu \mathrm{m}$ [Coyner, 1984]. Wissler [1987] measured the permeability of our sample.

Another clean quartz arenite is the Flathead sandstone of Wyoming, which 
contains about $98 \%$ quartz and about $2 \%$ clay, and has a porosity of about $10.6 \%$ [Caruso et al., 1985]. Grains are subrounded to rounded and poorly sorted, and have an average diameter of about $350 \mu \mathrm{m}$ [Caruso et al., 1985]. Permeability and formation factor for our sample were measured by Wissler [1987].

\section{Analysis}

For most formations we studied one sample, but for two formations we studied two samples, for a total of eight sandstone samples. Table 2 lists the number of low-magnification images analyzed for each sandstone sample. We examined two or three high-magnification images for all the sandstones, except that we used only one high-magnification image for one of the two ironton-Galesville sandstone samples. Representative low-magnification SEM images and associated correlation functions for each sample are shown in Figs. 12-19. Low magnifications (given in the figure captions) were between $21 \times$ and $100 \times$. Pixel sizes were typically $3-4 \mu \mathrm{m}$ for the low-magnification digital images.

We used the correlation function curves calculated for low-magnification images to finc the parameters $r_{c}$ and $r_{g}$ as well as the porosity for each of our eight natural sandstone samples. For each sample, correlation functions determined from at least two and, in some cases, five images were used. Results from this analysis are presented in Table 2 . We also give measured porosities that have been reported for these sandstones [Caruso et al., 1985; Daily and Lin, 1985; Wissler, 1987]. The computed porosities match the measured values to within a few percent for all samples, shown in Table 2.

The mean pore size parameter $r_{c}$ had values between 19 and $35 \mu \mathrm{m}$ for all samples except the Flathead sandstone. The uncertainties in the values shown in Table 2 were related in part to the number of images examined, with small variation observed for samples that were represented by only two images. We did, however, find that for samples represented by four or more images, the uncertainty appears to be related to the degree of sorting in the sample. The moderately well-sorted Berea sandstone sample Berq in Table 2 has smaller variation in the $r_{c}$ values determined using different images than was found for the poorly sorted IrontonGalesville sample \#775. A core-sized sample can be characterized best if four to five images are used. Fewer images may not provide enough statistical information, but more images are apparently unnecessary. The results shown in Table 2 suggest that we were able to determine the effective pore diameter to within at least a factor of 2 for all of our samples.

Table 2. Pore and grain parameters for natural sandstones.

\begin{tabular}{|c|c|c|c|c|c|c|c|c|}
\hline Sample & $\begin{array}{l}\text { No. of } \\
\text { Images }\end{array}$ & $\phi_{\text {obs }}$ & $\phi_{\text {calc }}$ & $\begin{array}{c}r_{c} \\
(\mu \mathrm{m})\end{array}$ & $\begin{array}{c}r_{g} \\
(\mu \mathrm{m})\end{array}$ & $\begin{array}{c}\text { Grain diam. } \\
(\mu \mathrm{m})\end{array}$ & $\stackrel{s}{\left(\mu m^{-1}\right)}$ & $\begin{array}{c}D_{H} \\
(\mu m)\end{array}$ \\
\hline I-G \#785 & 2 & b & 0.30 & $19 \pm 3$ & 50,120 & b & 0.1030 & 12.0 \\
\hline I-G \#775a & 4 & b & 0.28 & $29 \pm 18$ & $50-330$ & b & 0.1330 & 8.4 \\
\hline Berea Berq & 5 & $0.15-0.19^{c-f}$ & 0.23 & $20 \pm 10$ & $60-100$ & $100-120^{c, d}$ & 0.1072 & 8.6 \\
\hline Frontier & 2 & $0.217^{d, f}$ & 0.19 & $27 \pm 4$ & $65-120$ & $130 \mathrm{~d}$ & 0.0913 & 8.3 \\
\hline Berea BF1 & 2 & $0.153^{e}$ & 0.16 & $20 \pm 2$ & $80-170$ & $100-120^{c, d}$ & 0.1133 & 5.6 \\
\hline Tensleep & 2 & $0.181^{d, f}$ & 0.15 & $30 \pm 8$ & $60-220$ & $115-170^{d}$ & 0.0756 & 7.9 \\
\hline Navajo & 3 & $0.138^{c}$ & 0.15 & $35 \pm 12$ & $100-220$ & $150^{c}$ & 0.0900 & 6.7 \\
\hline Flathead & 3 & $0.106^{\mathrm{d}, \mathrm{f}}$ & 0.09 & $76 \pm 8$ & $160-220$ & $350^{d}$ & 0.0797 & 4.5 \\
\hline
\end{tabular}

I-G signifies Ironton-Galesville sandstorie.

bNot available.

'From Coyner [1984].

dFrom Caruso et al. [1985].

'From Daily and Lin [1985].

fFrom Wissler [1987]. 
(a) 785

$\bigvee_{100 \mu \mathrm{m}}$

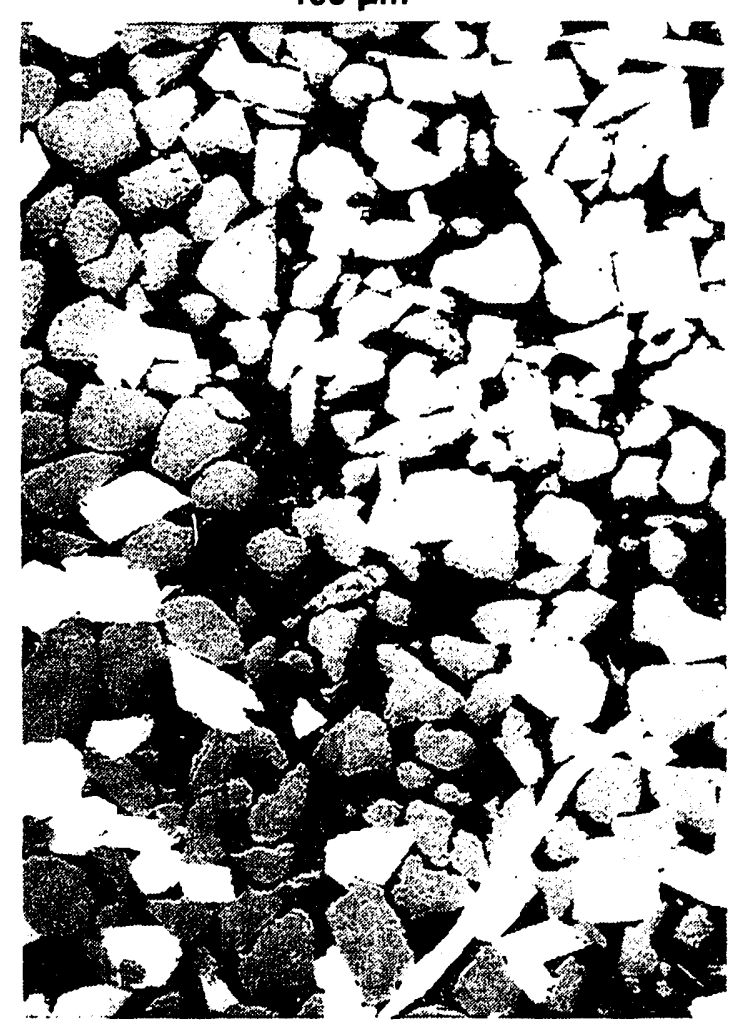

(b)

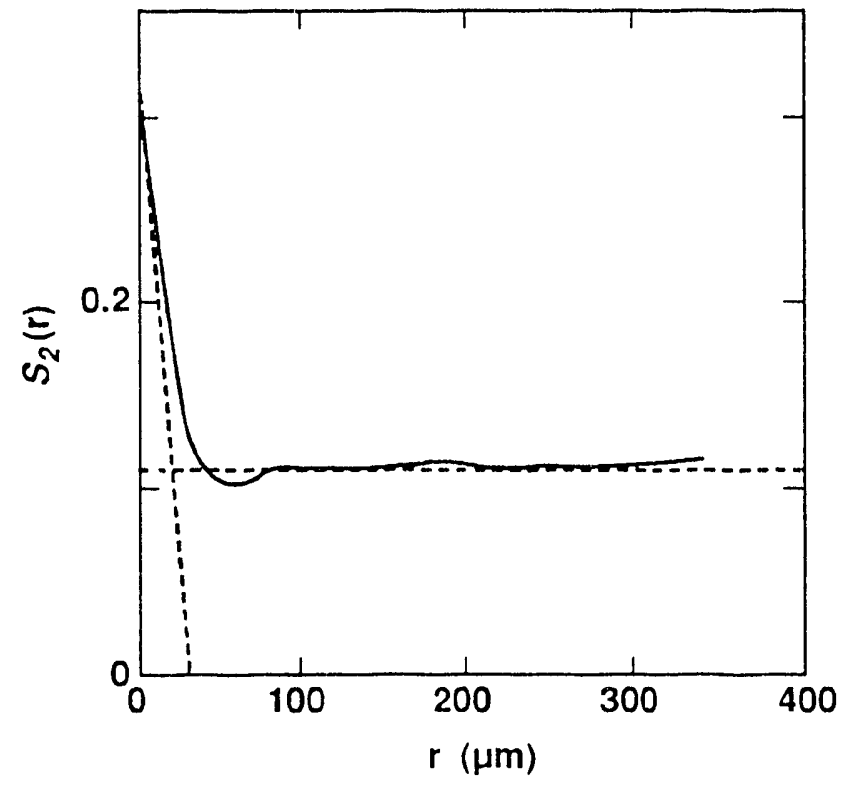

Figure 12. (a) $100 \times$ magnification SEM image [after Berryman and Blair, 1986] and (b) related correlation function (solid curve) for IrontonGalesville sandstone sample \#785. Slope near the origin is indicated by a dashed line. (a) 775

$\left.\right|_{100 \mu \mathrm{m}} 100 x$

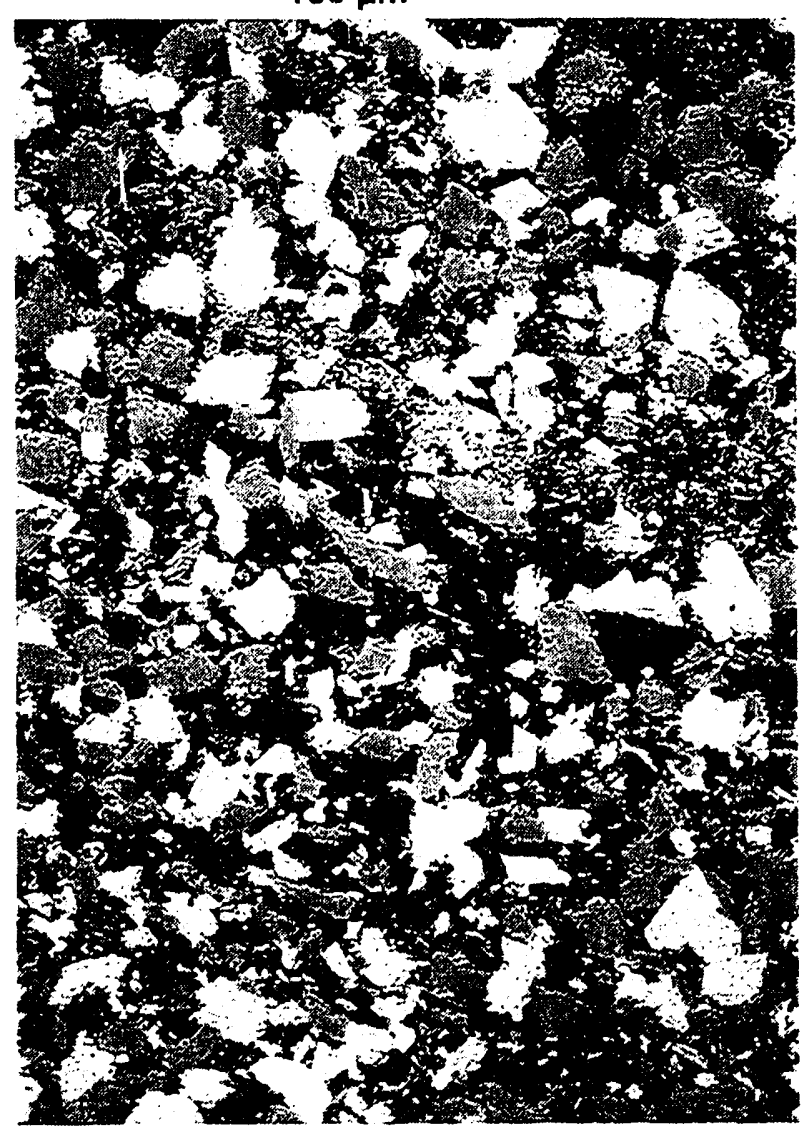

(b)

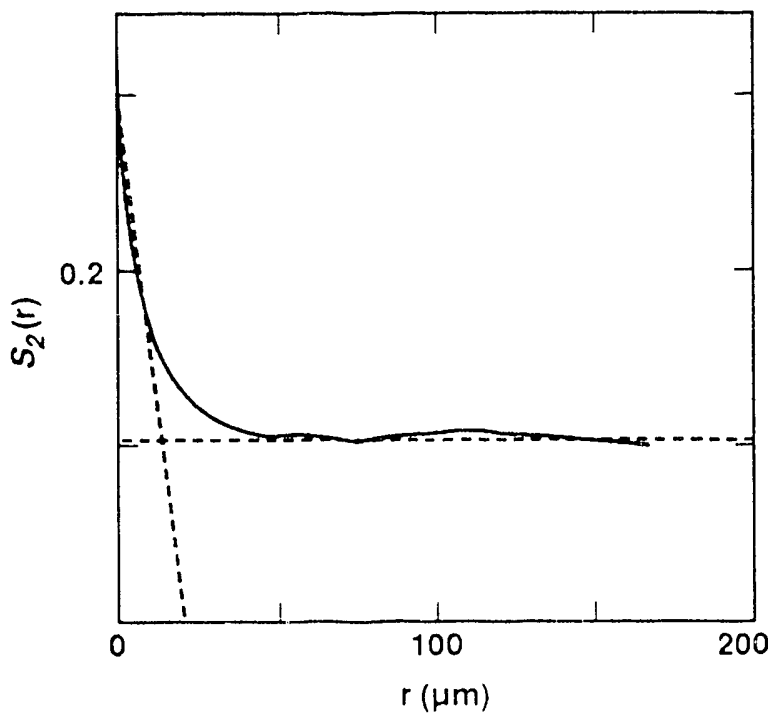

Figure 13. (a) $100 \times$ magnification SEM image [after Berryman and Blair, 1986] and (b) related correlation function (solid curve) for IrontonGalesville sandstone sample \#775. Slope near the origin is indicated by a dashed line. 
(a) Berq

$54 \times$

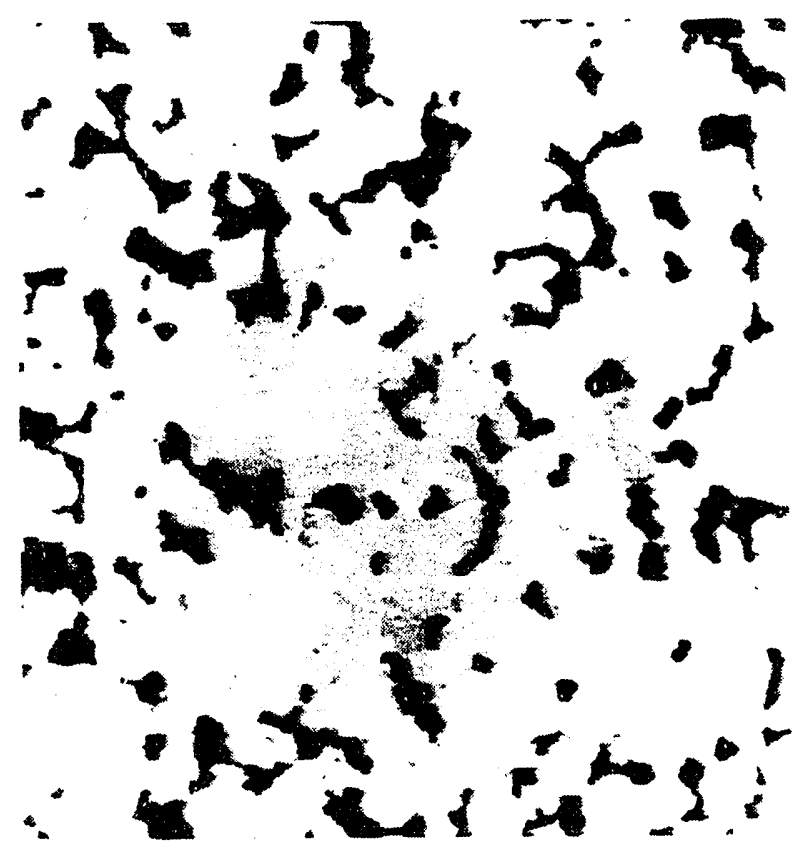

(b)

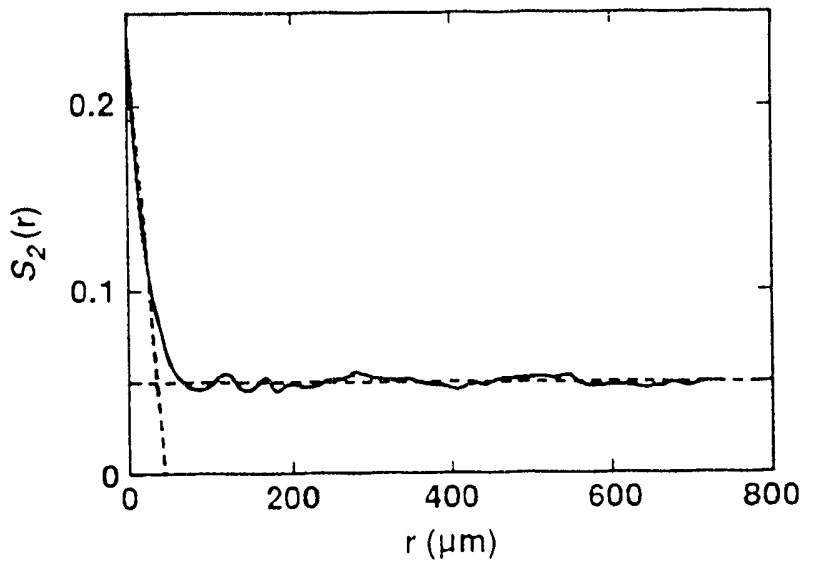

Figure 14. (a) $54 \times$ magnification SEM image and (b) related correlation function (solid curve) for Berea sandstone sample Berq [after Blair and Berryman, 1991,1992]. Slope near origin is indicated by dashed line. (a) FTR

$\underbrace{}_{100 \mu \mathrm{m}} 100 x$

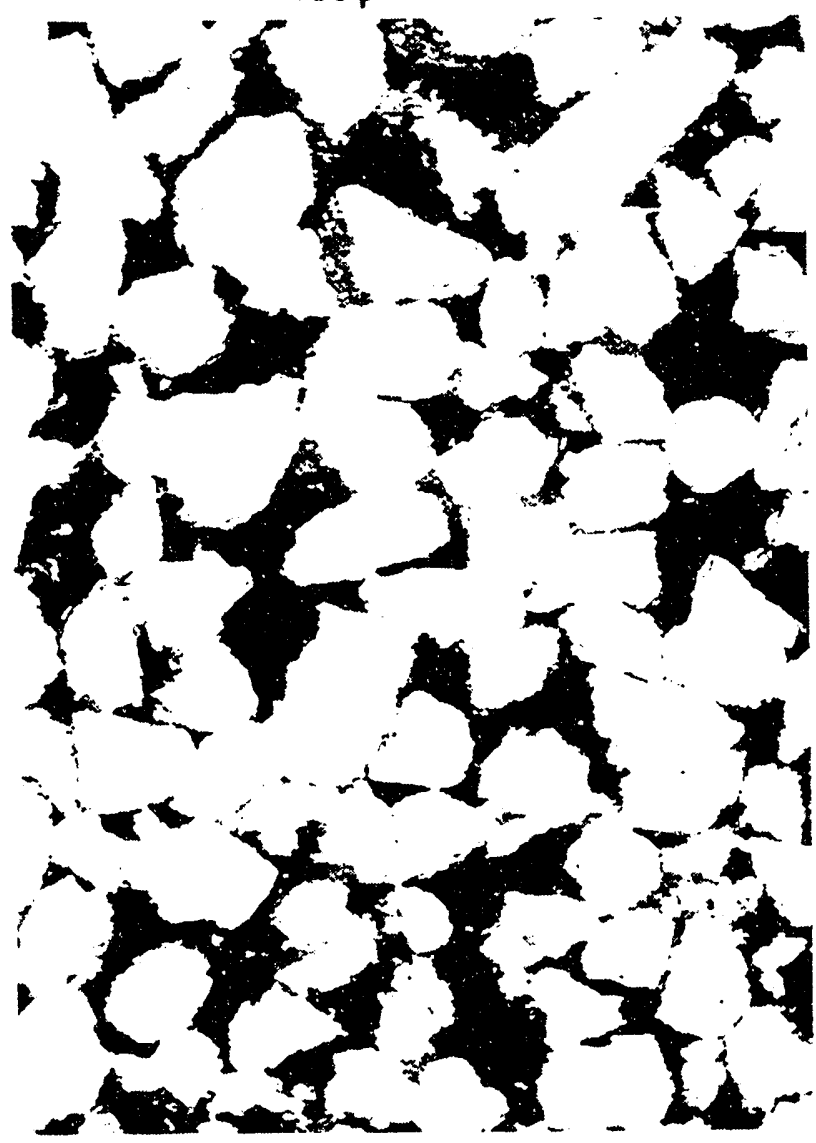

(b)

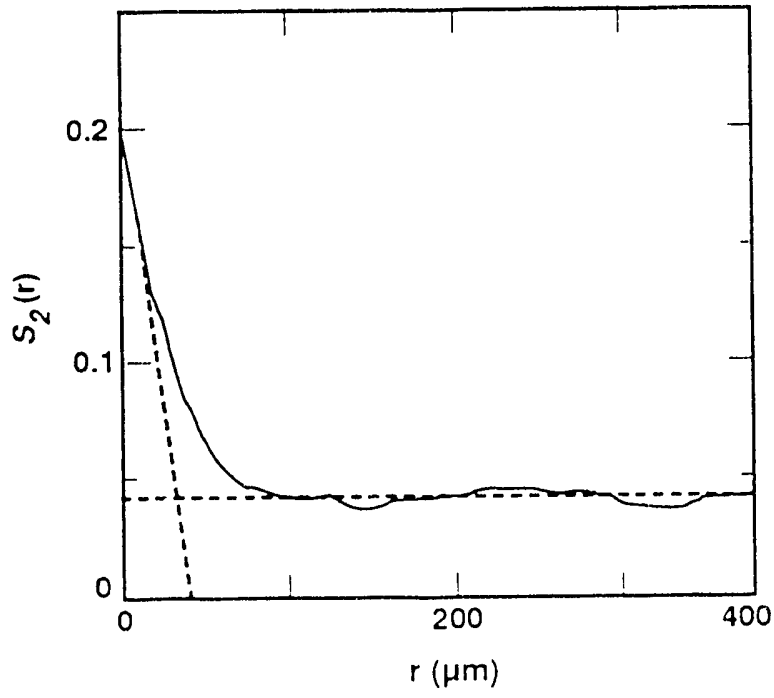

Figure 15. (a) $100 \times$ magnification SEM image and (b) related correlation function (solid curve) for Frontier sandstone. Slope near the origin is indicated by a dashed line. 


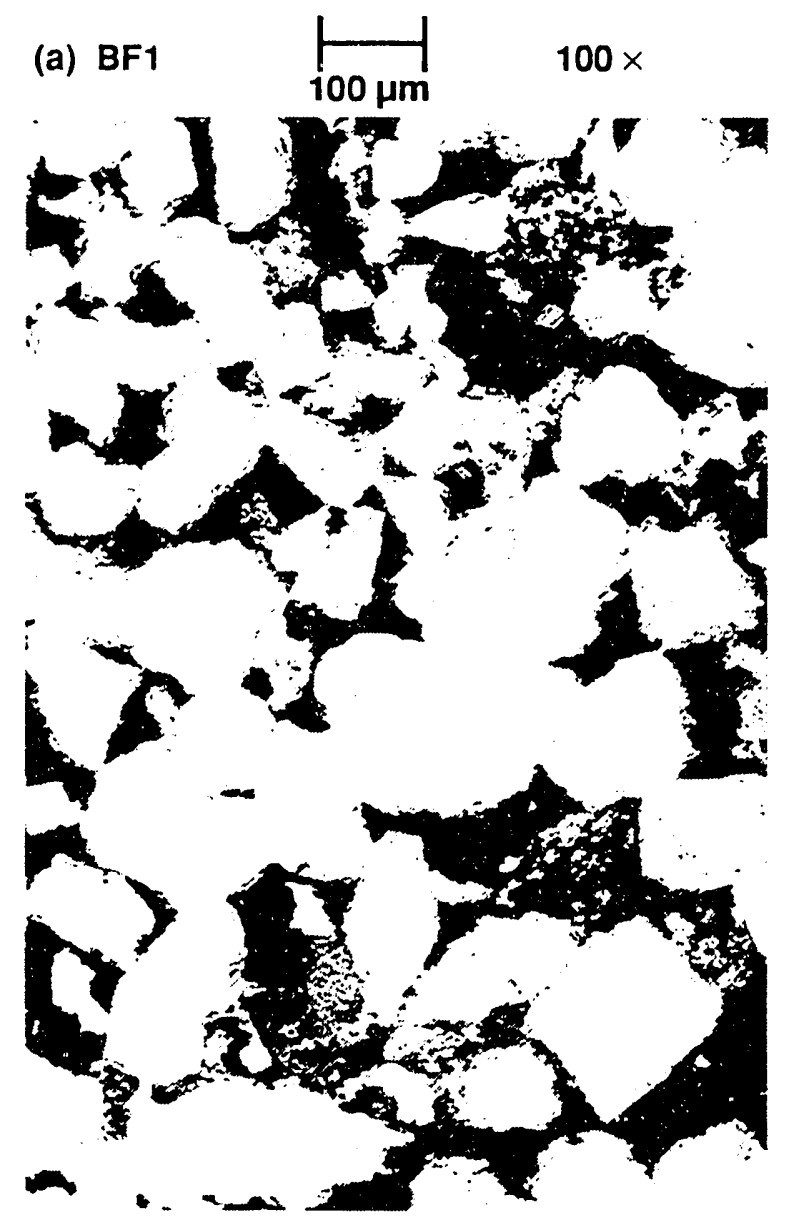

(b)

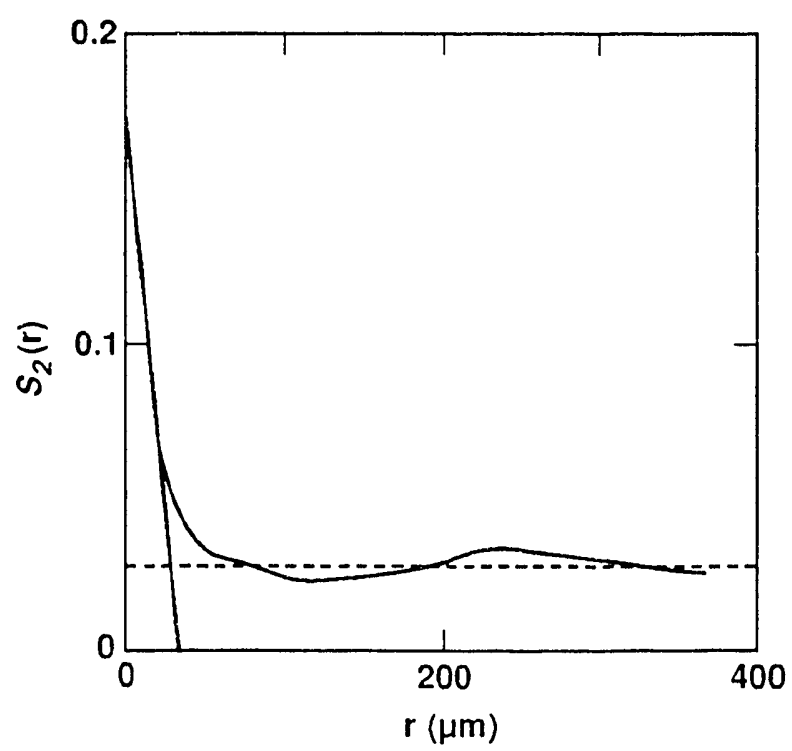

Figure 16. (a) $100 \times$ magnification SEM image [after Berryman and Blair, 1986] and (b) related correlation function (solid curve) for Berea sandstone sample BF1. Slope near the origin is indicated by a dashed line.

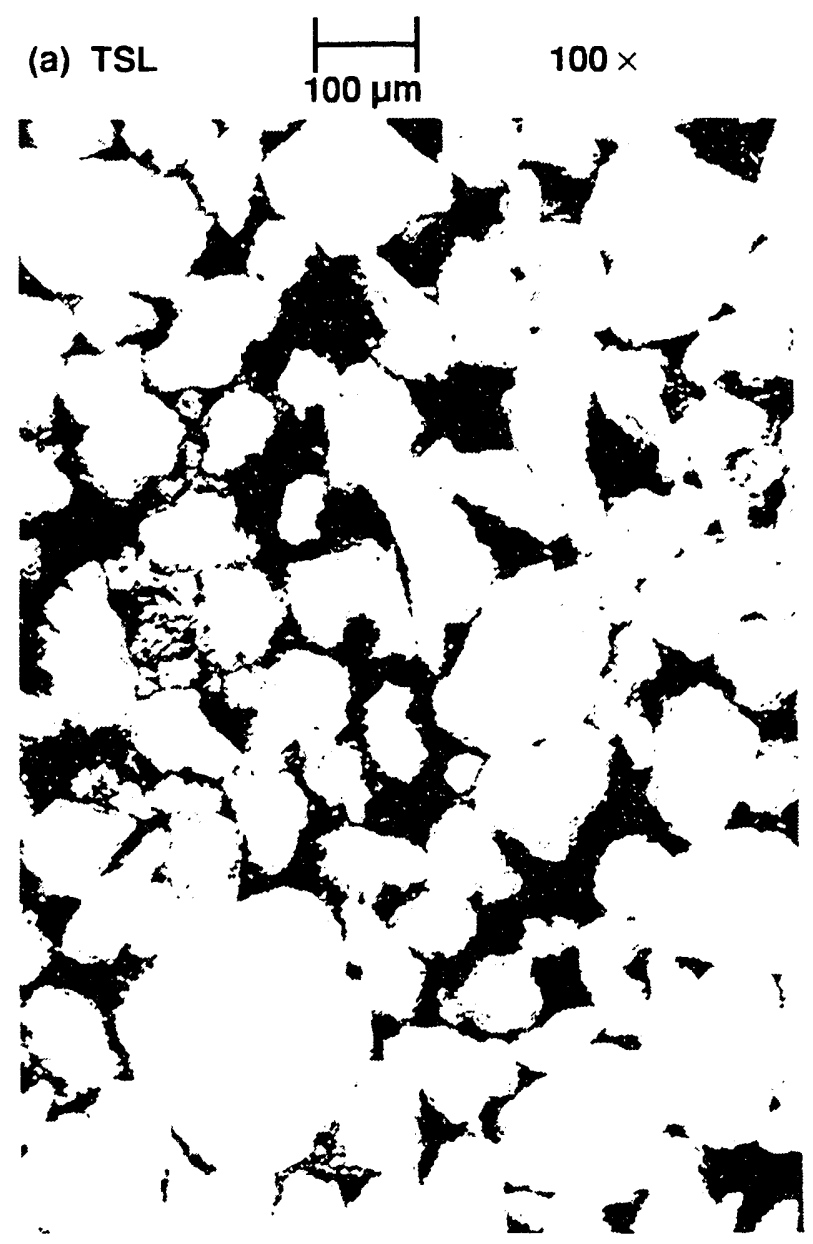

(b)

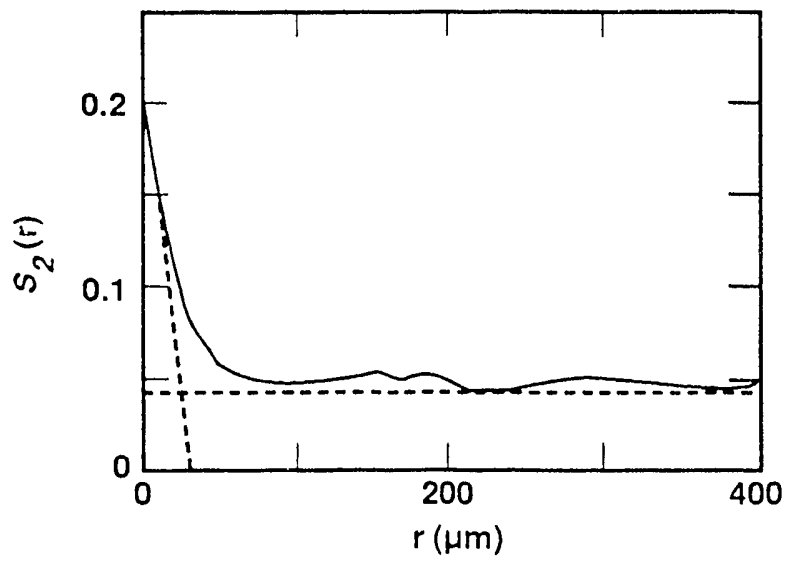

Figure 17. (a) $100 \times$ magnification SEM image and (b) related correlation function (solid curve) for Tensleep sandstone. Slope near the origin is indicated by a dashed line. 


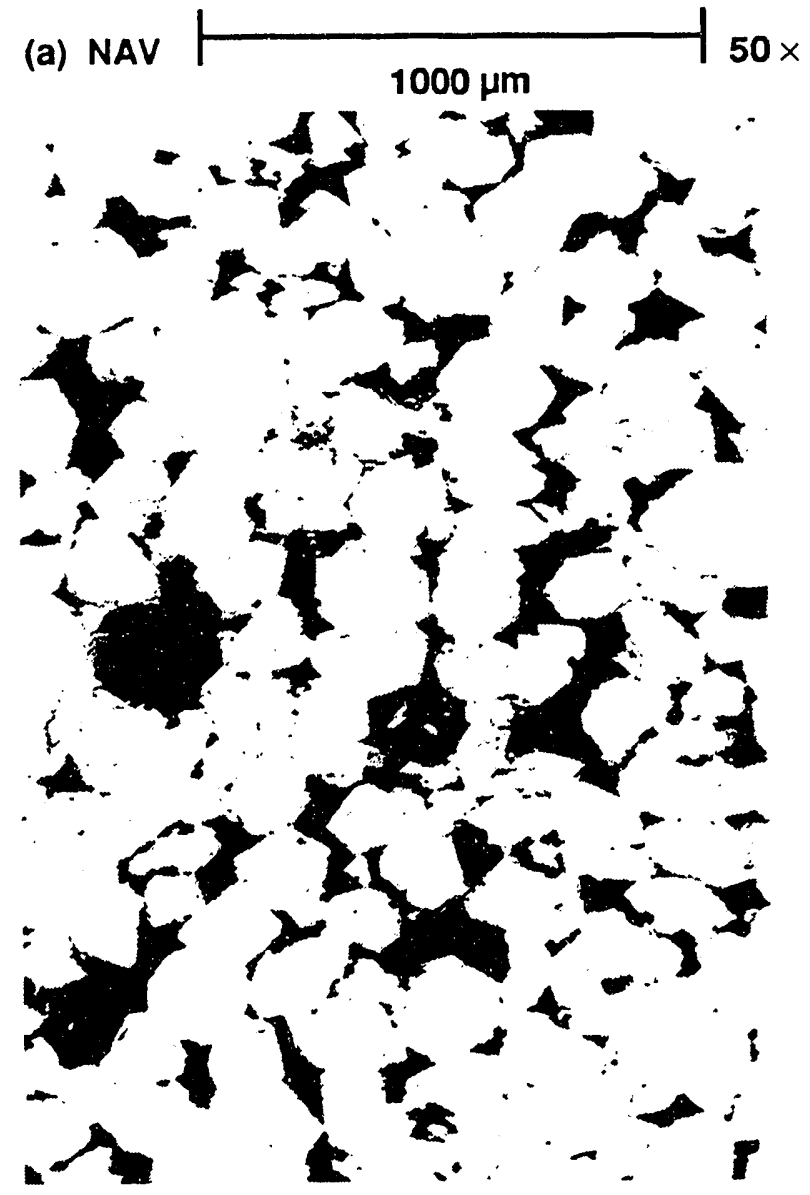

(a) FLT

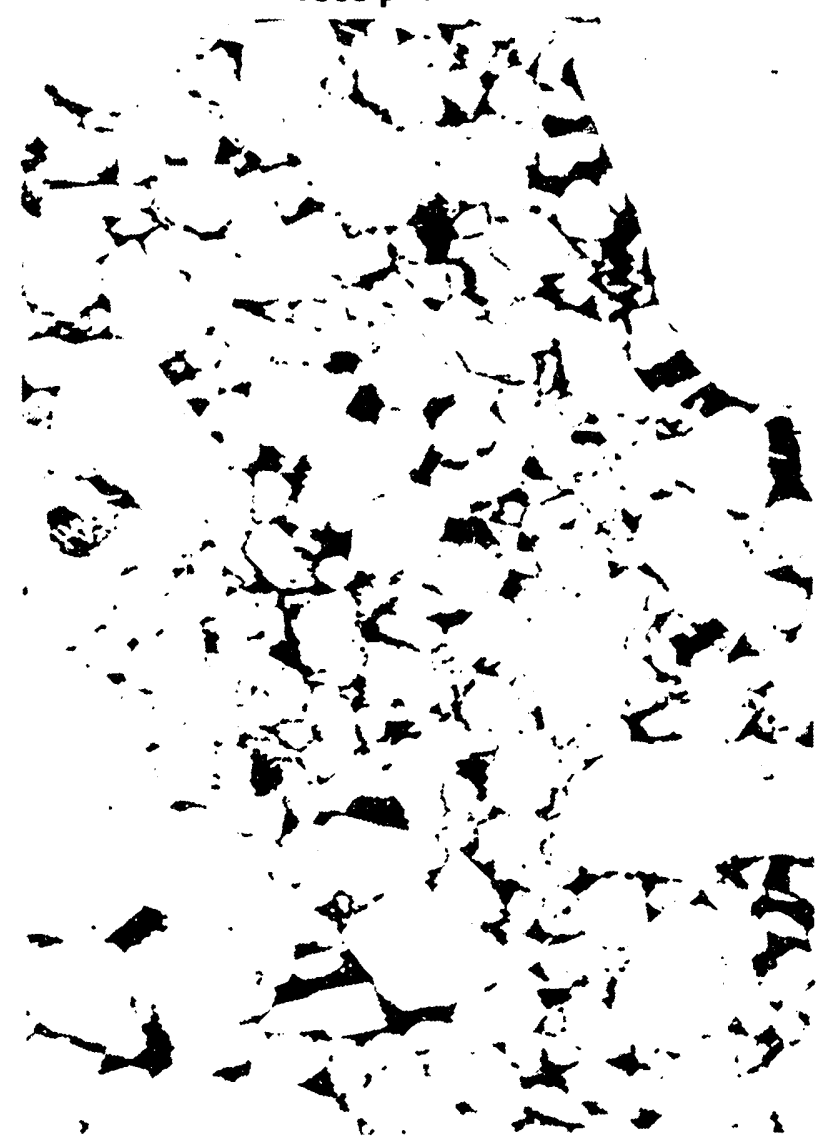

(b)

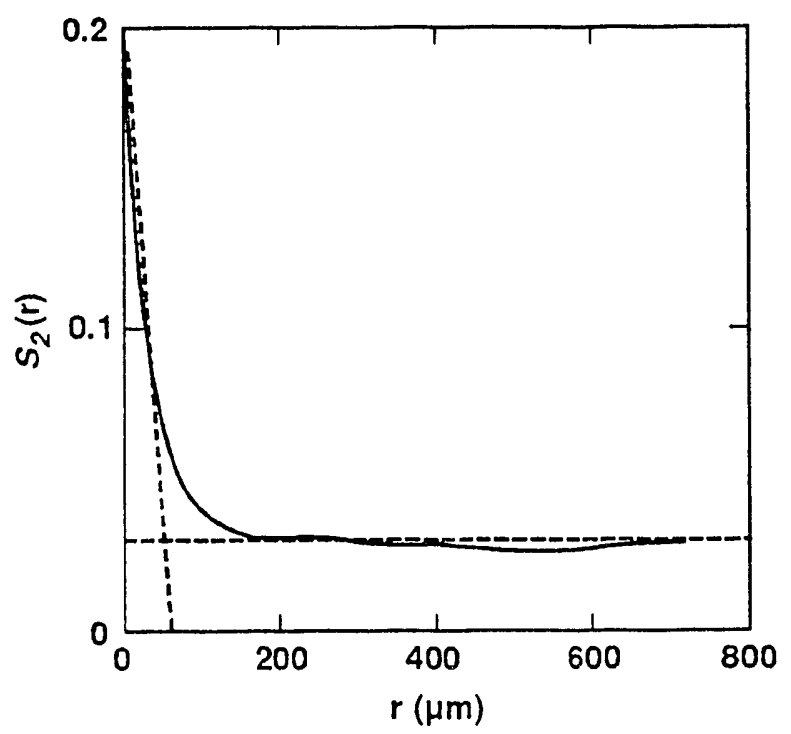

Figure 18. (a) $50 \times$ magnification SEM image and (b) related correlation function (solid curve) for Navajo sandstone. Slope near the origin is indicated by a dashed line.

(b)

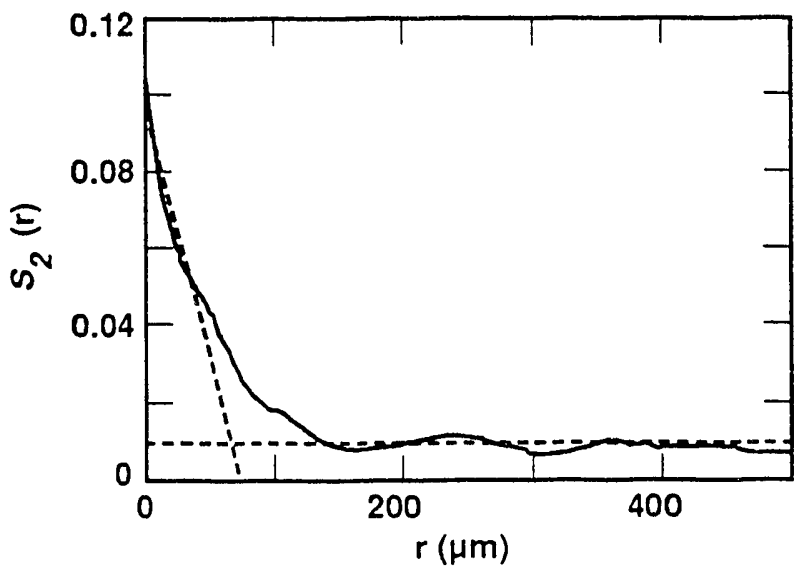

Figure 19. (a) $21 \times$ magnification SEM image and (b) related correlation function (solid curve) for Flathead sandstone. Slope near the origin is indicated by a dashed line. 
The values of $r_{g}$ listed in Table 2 are for minimum effective grain sizes as determined using the number of images given in the table. Most of the $r_{g}$ values listed in Table 2 were picked using the first distinct minimum in the $\mathrm{S}_{2}$ curve.

Table 2 presents results for two samples of Ironton-Galesville sandstone. Figure 12(a) shows a raw image of our Ironton-Galesville sandstone sample \#785. This figure shows the grains of this sandstone to be discrete, which is reflected in the two-point correlation function for this sandstone [Fig. 12(b)]. The $r_{g}$ value was determined from a distinct minimum in the $S_{2}$ curve at about $50 \mu \mathrm{m}$, while the $r_{c}$ value indicates an effective pore diameter of $19 \pm 3 \mu \mathrm{m}$. At correlation lengths greater than $100 \mu \mathrm{m}$, the lack of oscillations in the $S_{2}$ curve indicates that particles are not spherical, and the material microgeometry is uncorrelated at these lengths.

An image of our Ironton-Galesville sandstone sample \#775 is shown ir. Fig. 13(a). This image is much different from the image for sample \#785 [Fig. 12(a)]. The grains are more poorly sorted, and a zone of fine-grained material occurs in the top half of the image. The corresponding $S_{2}$ curve shown in Fig. 13(b) is indicative of the poorly sorted structure, as no clear minimum is present and the function gradually approaches the $\phi^{2}$ value, having $r_{g}$ near $50 \mu \mathrm{m} . S_{2}$ curves computed for other images of this sample also illustrated its poorly sorted nature, and different magnifications of $20 \times$ and $100 \times$ produced a range of estimates for the $r_{g}$ value (Table 2). Different magnifications emphasize the importance of different grain sizes in poorly sorted materials. Our findings for this sample of the Ironton-Galesville sandstone are consistent with the description of Walton [1981], which states that the sandstone is poorly sorted and has silty, clayey zones and interbedded shale.

Given the differences in images and correlation functions for these samples, note that the effective pore- and grain-size estimates shown in Table 2 for samples \#775 and \#785 of the Ironton-Galesville sandstone are similar. A slightly higher $r_{c}$ estimate was found for sample \#775 than for \#785. However, more images of \#785 would be required to verify this observation. Sample \#775 contains more large grains, possibly because it was represented by some images having lower magnifications than the $100 \times$ used for sample $\# 785$.

Images and correlation function curves for our two Berea sandstone samples are shown in Figs. 14 and 16. The sample shown in Fig. 14 is well sorted, while the sample shown in Fig. 16 contains some fine-grained material along with sand grains. This difference in sorting is reflected in the $S_{2}$ functions computed for these images, shown in Figs. 14(b) and 16(b). The curve in Fig. 14(b) shows an $r_{g}$ minimum at about $80 \mu \mathrm{m}$ followed by oscillations resembling the curve for the ideal hard sphere model, while $S_{2}$ in Fig. 16(b) gradually decreases to a minimum between 80 and $120 \mu \mathrm{m}$. The shape of the curve in Fig. 16(b) may reflect the occurence of a wider grain size distribution and more angular grains in this sample. Values of $r_{C}$ determined for the two samples show good agreement and indicate an effective pore diameter of $20 \mu \mathrm{m}$. The $\mathrm{r}_{g}$ minimum value of $60-100 \mu \mathrm{m}$ for Berea sandstone sample Berq is in excellent agreement with grain-size estimates that Caruso et al. [1985] determined using a point-counting technique (Table 2). Although the porosities of our two Berea samples were quite different, the effective pore and grain sizes were similar. . Since the Berea sandstone is a moderately wellsorted clean quartz arenite, our analysis found much better agreement for parameters describing the two different samples than we found when analyzing the two Ironton-Galesville sandstone samples.

The Frontier sandstone in Fig. 15 is also a clean quartz arenite, with well-sorted grains. The discrete nature and uniform size of the grains can be seen in Fig. 15(a) and are indicated in the $S_{2}$ function shown in Fig. 15(b). We estimated that the effective pore diameter for this sandstone is about $27 \mu \mathrm{m}$. The minimum near $65-120 \mu \mathrm{m}$ taken as $r_{g}$ is in good agreement with the $130-\mu \mathrm{m}$ grain diameter found by Caruso et al. [1985]. The $S_{2}$ curve does not show distinct oscillations because the grains in this sandstone are more angular than round. Our analysis shows that this sandstone is similar to the Berea sandstone.

The Tensleep sandstone shown in Fig. 17 had an effective pore diameter of about $30 \mu \mathrm{m}$. This clean quartz arenite is well sorted, but grain-size estimates reported by Caruso et al. [1985] varied 
for different samples (see Table 2). We found the $S_{2}$ curve minimum for $\mathrm{r}_{\mathrm{g}}$ at about $60 \mu \mathrm{m}$, in agreement with the grain-diameter values given by Caruso et al. [1985].

A low-magnification SEM image for a sample of Navajo sandstone is shown together with the associated correlation function (Fig. 18). Very few discrete grains are visible in this image, as the grains appear to form large aggregates with mean size several times larger than that of a single grain. This morphology is reflected in the two-point correlation function, which gradually approaches the asymptotic $\phi^{2}$ value at a lag of about $220 \mu \mathrm{m}$. Even though the Navajo sandston $€$ is well sorted, there is some heterogeneity in grain size and shape, and the presence of grain aggregates complicates the analysis.

The $r_{g}$ minimum in Fig. 18(b) at about $220 \mu \mathrm{m}$ provides an estimate of the size of the grains and is in good agreement with the grain diameter that Coyner [1984] reported for Navajo sandstone (Table 2). We measured an $r_{c}$ value indicating an effective pore diameter of $35 \pm 12 \mu \mathrm{m}$.

The Flathead sandstone shown in Fig. 19(a) is poorly sorted and has grain sizes spanning three orders of magnitude. The correlation function for the sample in Fig. 19(b) shows an $\mathrm{r}_{\mathrm{g}}$ minimum at about $160 \mu \mathrm{m}$, followed by oscillatory behavior that indicates the grains are rounded rather than angular. The roughness of the curve at lags of about 0 to $100 \mu \mathrm{m}$ may be related to the poor sorting. The $r_{g}$ value gives a grain size estimate that is somewhat smaller than the $350-\mu \mathrm{m}$ diameter reported by Caruso et al. [1985]. Larger grains apparent on some of our images could not be measured at 21-100x magnifications, since the complete correlation function can only be computed accurately for objects having dimensions up to half of the SEM image diameter [Berryman, 1985b].

The $r_{c}$ value of $76 \mu \mathrm{m}$ measured for the Flathead sandstone is much higher than the values determined for our other natural sandstone samples. This is a consequence of the low porosity of the Flathead sandstone. Physically, this can be interpreted as a result of having a larger percentage of big pores in a lowporosity rock. The contribution of a few large pores can dominate the effective pore diameter measured using $r_{c}$ for a low-porosity rock. The poorly sorted fabric of the Flathead sandstone may also contribute to finding a large pore size.

We used larger magnification images, such as those shown in Figs. 20-27, to find the slope of $S_{2}$ near the origin and to compute $s$ for each of the natural sandstones. Míagnifications were $490-1000 \times$ for the high-resolution images of the natural sandstones. The image specific-surface areas determined for all the natural sandstone samples are listed in Table 2, together with the hydraulic diameter values calculated using Eq. (12).

The high-magnification $S_{2}$ curves (Figs. 2027) show that for all sandstones, features with sizes smaller than $3 \mu \mathrm{m}$ control the value of the image specific surface. The slope of the curve changes only a few micrometers from the origin in each of these figures. For the Frontier and Navajo sandstones, features with sizes near $1 \mu \mathrm{m}$ control the image specific surface. We found that, for all rocks, the size determined from the break in the $S_{2}$ curve was independent of magnification when magnification ranged from 450-1200x.

In Table 2 we see that the image specific surface was in the range of $0.0756-0.1330 \mu \mathrm{m}^{-1}$ for all rock samples. This is a rather narrow range, but even though the values appear similar, the permeability computed using Eq. (14) depends on the square of $s$, which magnifies the effect of different values for $s$. The glass beads had smaller $s$ values of about $0.02 \mu^{-1}$. The rougher surfaces of the natural sand grains caused generally higher values of $s$ than were observed for the smooth glass beads.

Because the image specific-surface values were all approximately the same for the rocks in Table 2, the hydraulic diameter values varied mainly with the porosities. The hydraulic diameter values in Table 2 are nearly an order of magnitude smaller than the values for the synthetic sandstone samples shown in Table 1. Comparison of synthetic and natural sandstone samples having similar porosities shows that the differences in the hyclraulic diameters are due to the differences in image specific-surface areas for sand grains and glass beads. 

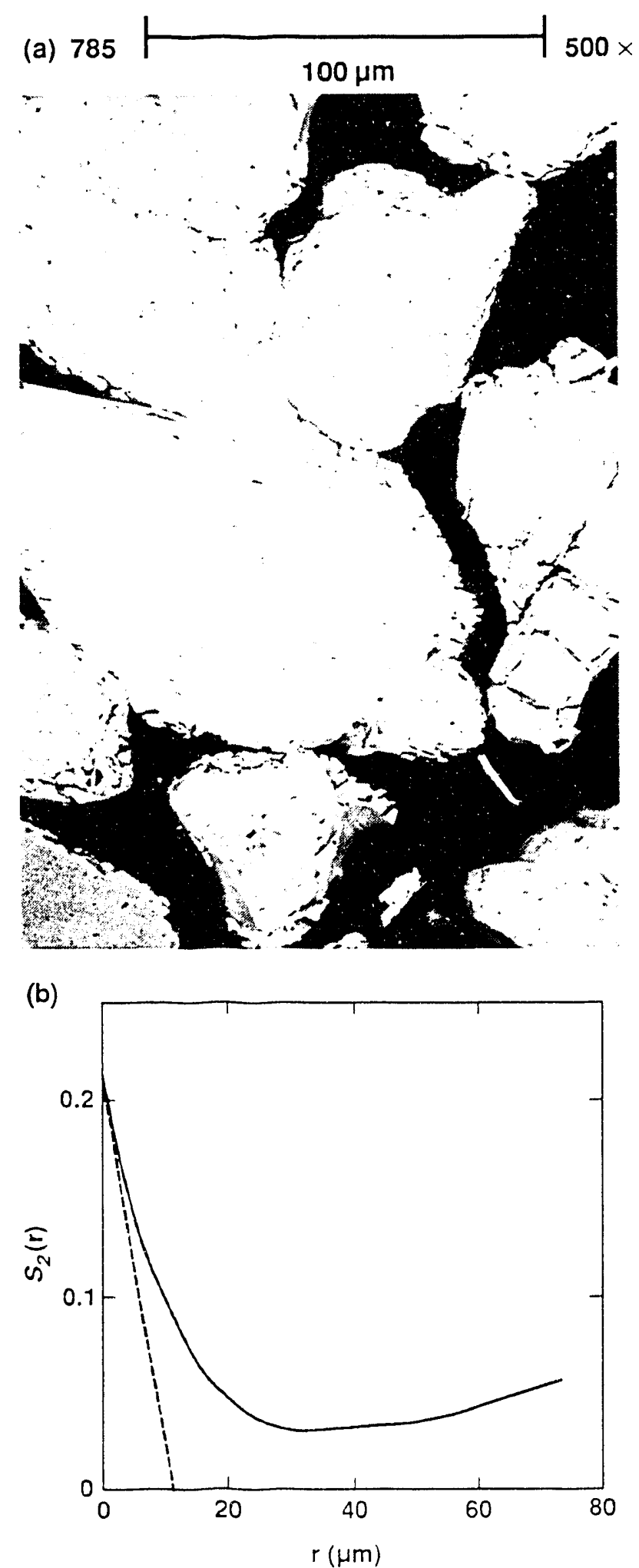

Figure 20. (a) $500 \times$ magnification SEM image and (b) related correlation function (solid curve) for Ironton-Galesville sandstone sample \#785. Slope near the origin is indicated by a dashed line.
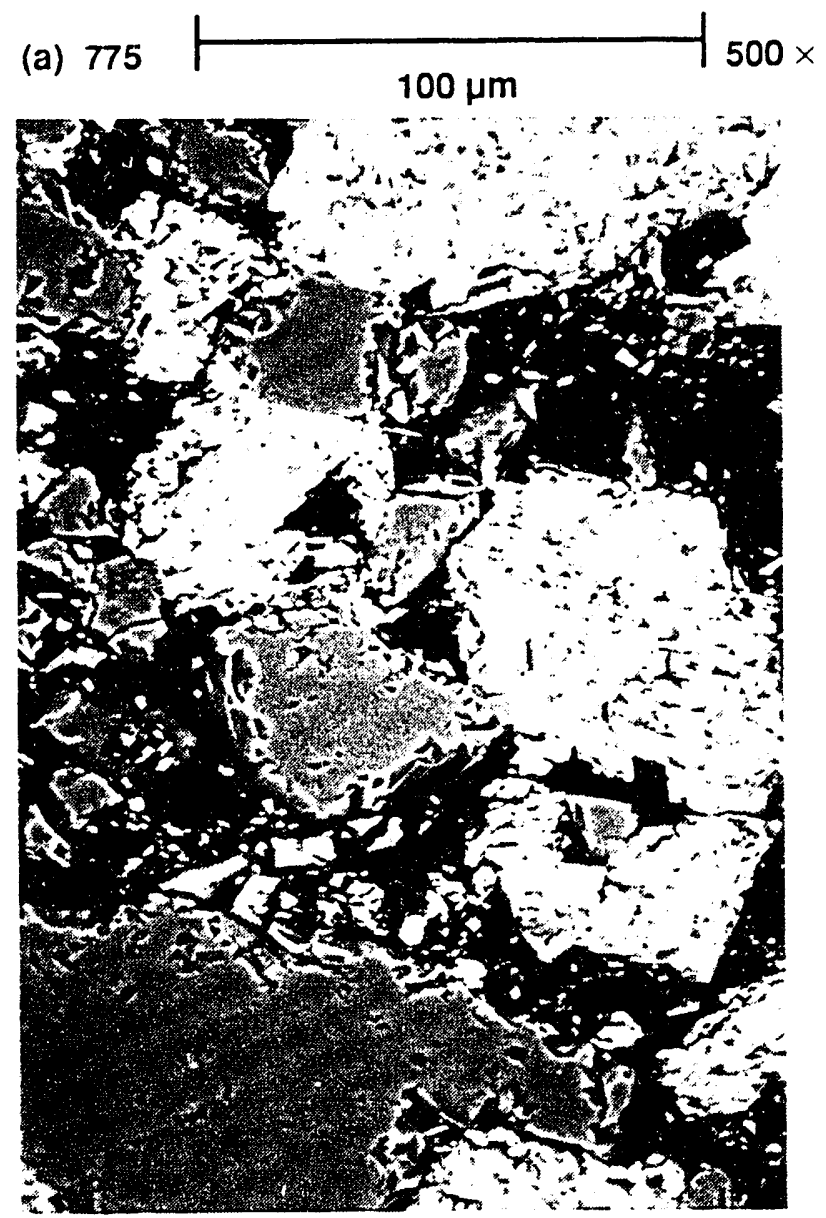

(b)

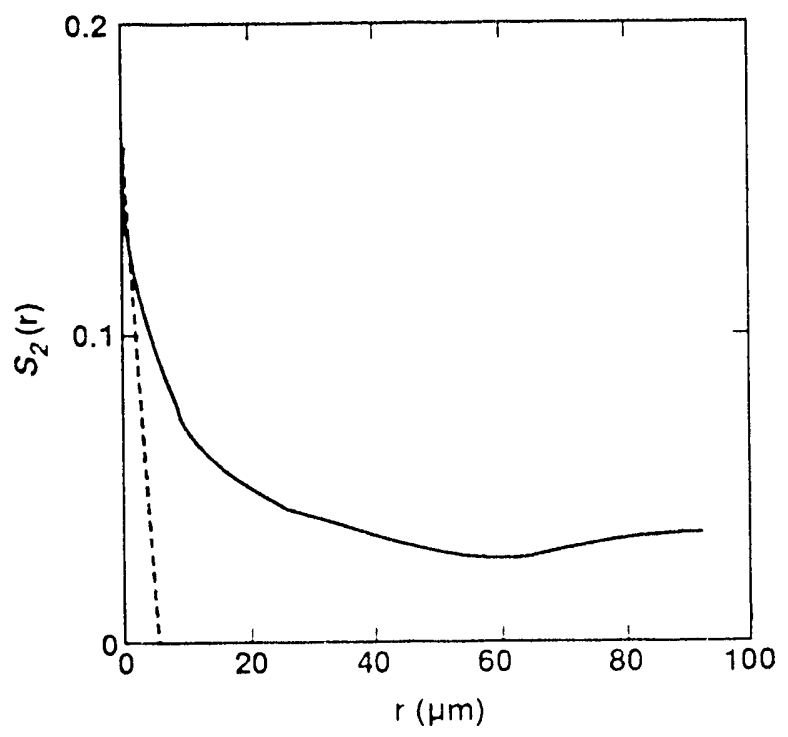

Figure 21. (a) $500 \times$ magnification SEM image and (b) related correlation function (solid curve) for Ironton-Galesville sandstone sample \#775. Slope near the origin is indicated by a dashed line. 
(a) Berq

$540 \times$

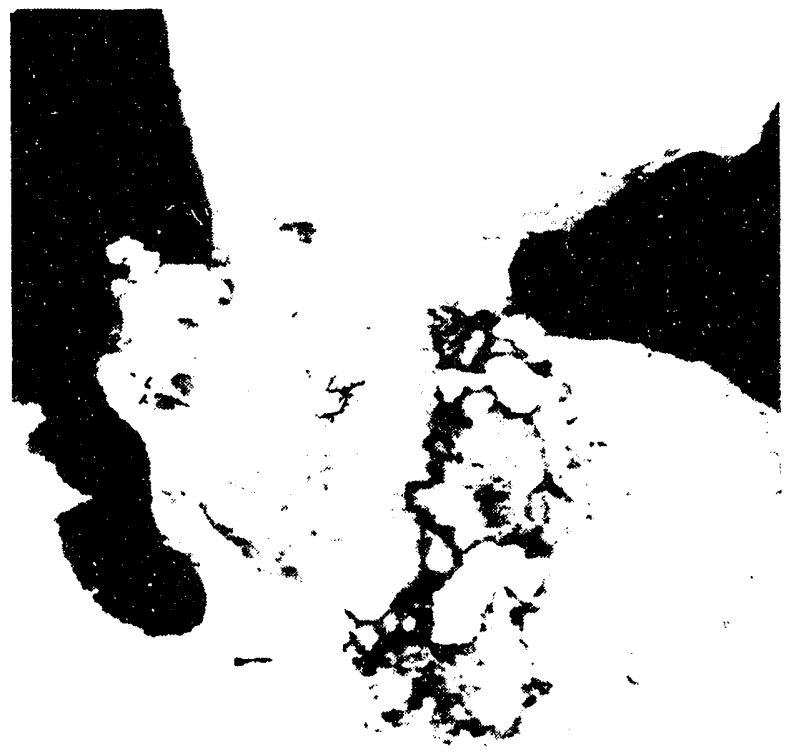

(b)

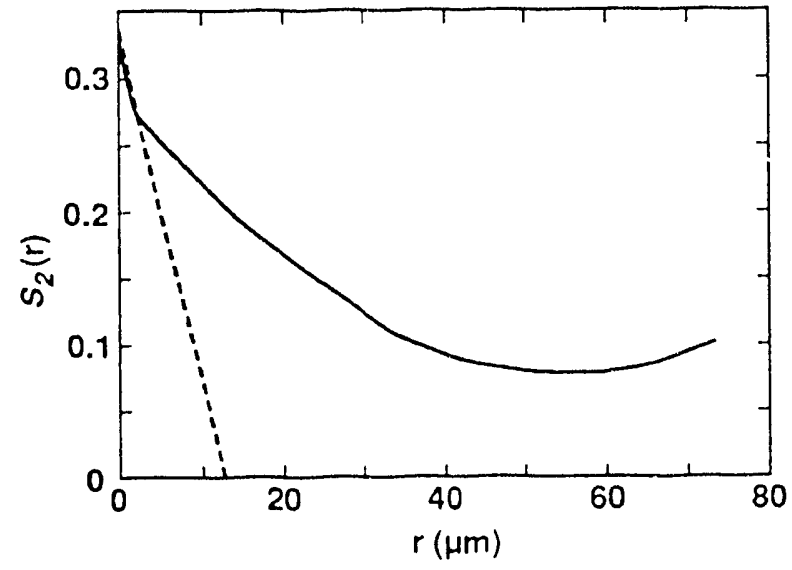

Figure 22. (a) $540 \times$ magnification SEM image and (b) related correlation function (solid curve) for Berea sandstone sample Berq [after Blair and Berryman, 1991,1992]. Slope near the origin is indicated by a dashed line.
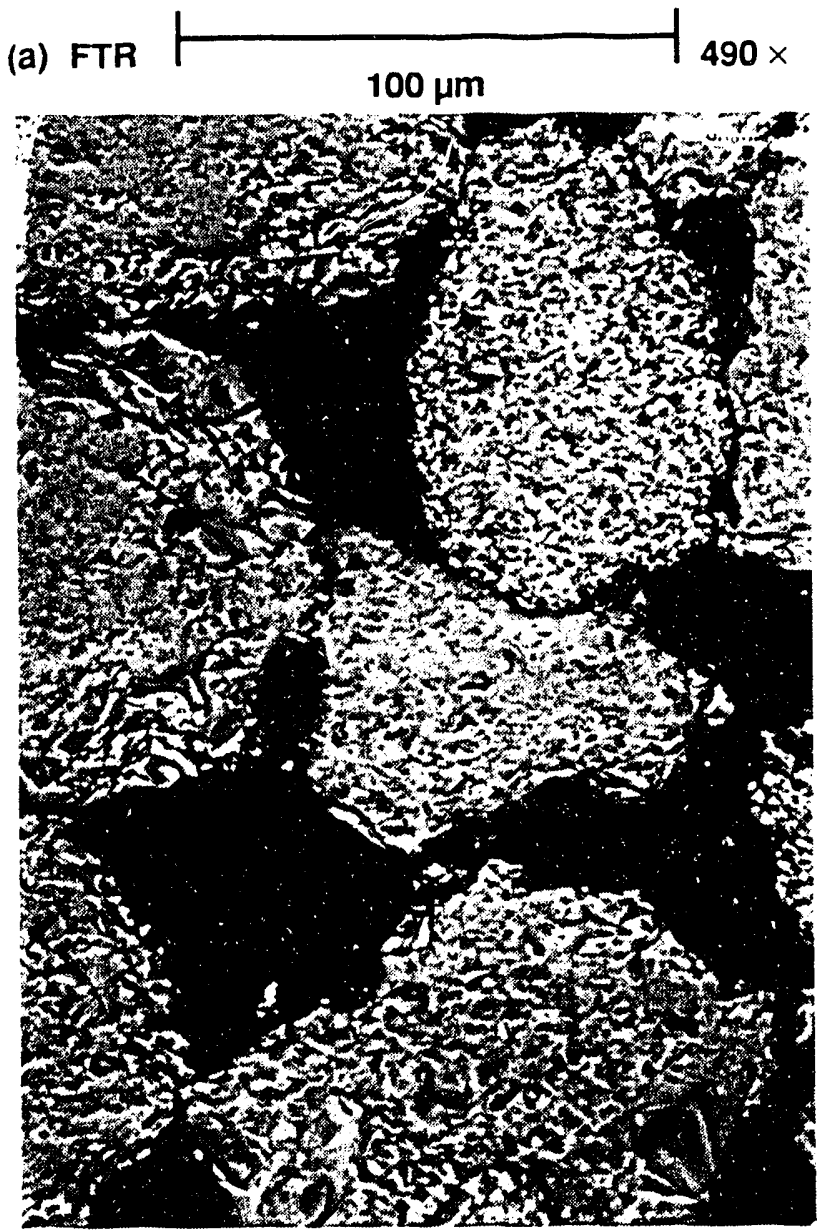

(b)

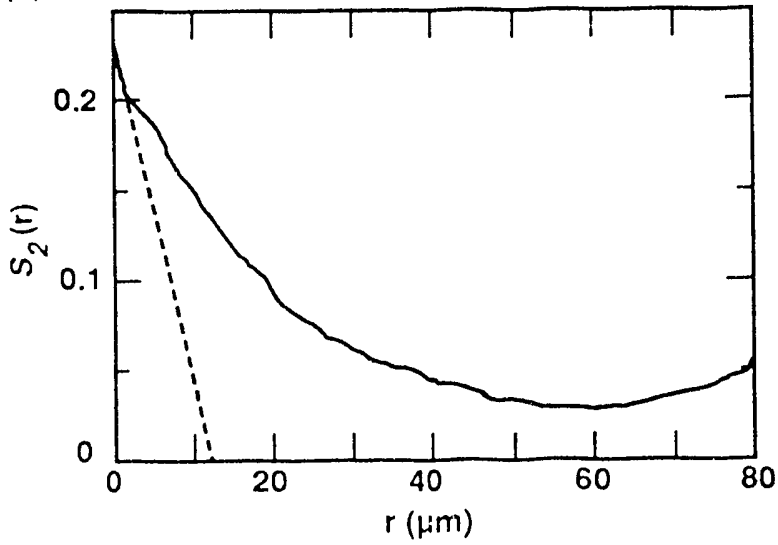

Figure 23. (a) $490 \times$ magnification SEM image and (b) related correlation function (solid curve) for Frontier sandstone. Slope near the origin is indicated by a dashed line. 
(a) BF1
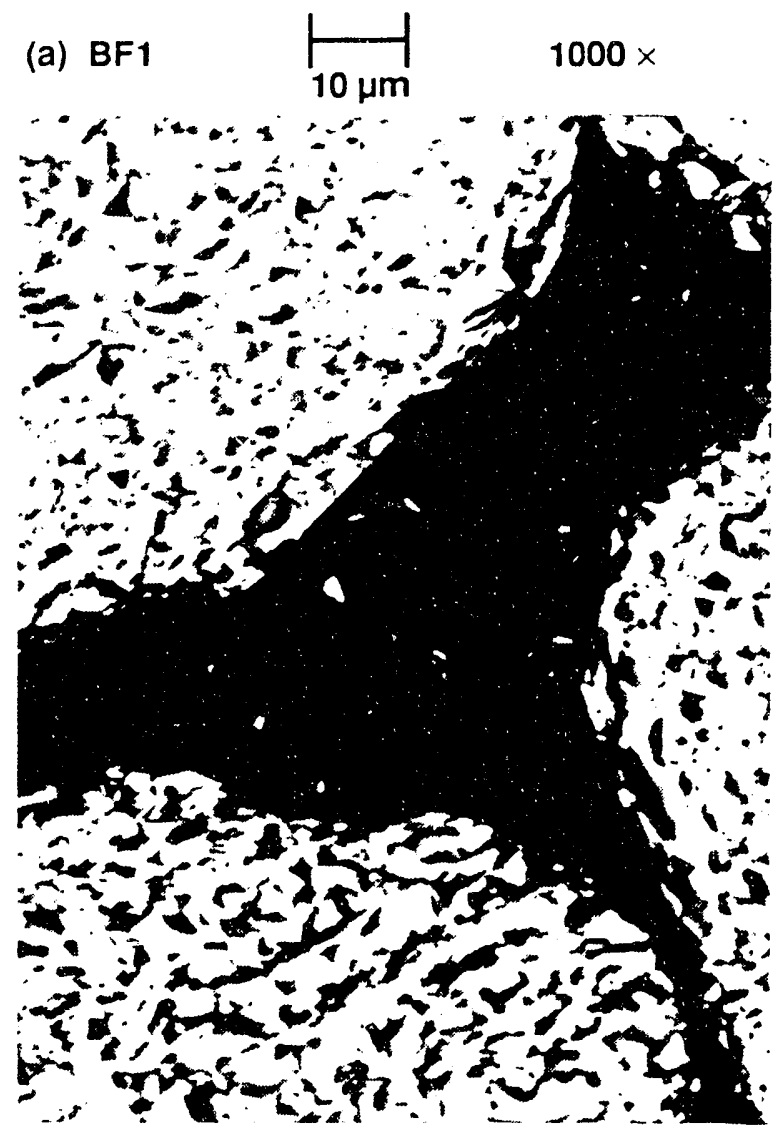

(b)

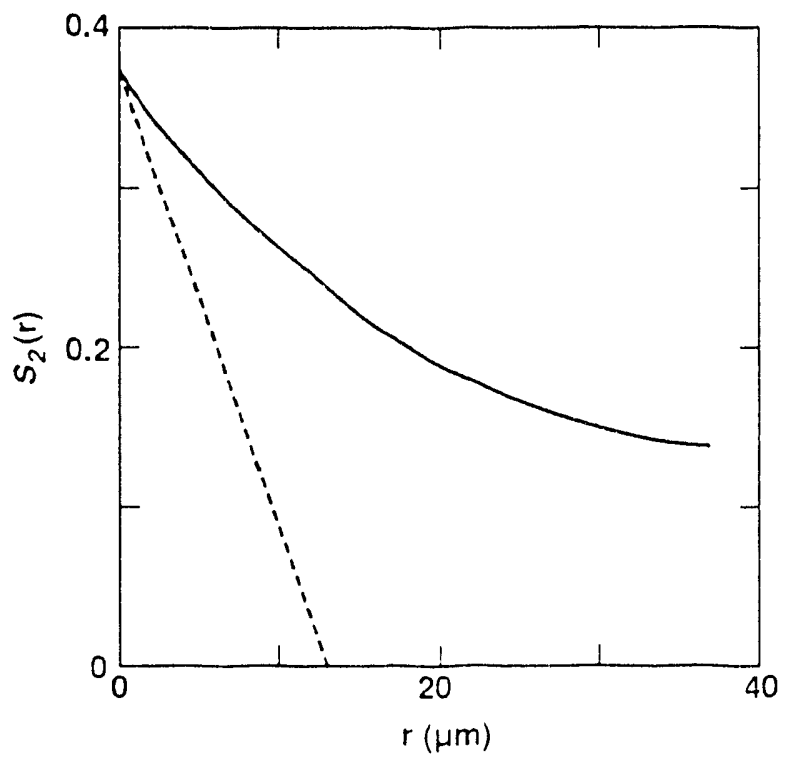

Figure 24. (a) $1000 \times$ magnification SEM image [after Berryman and Blair, 1986] and (b) related correlation function (solid curve) for Berea sandstone sample BF1. Slope near the origin is indicated by a dashed line. (a) TSL $100 \mu \mathrm{m}$
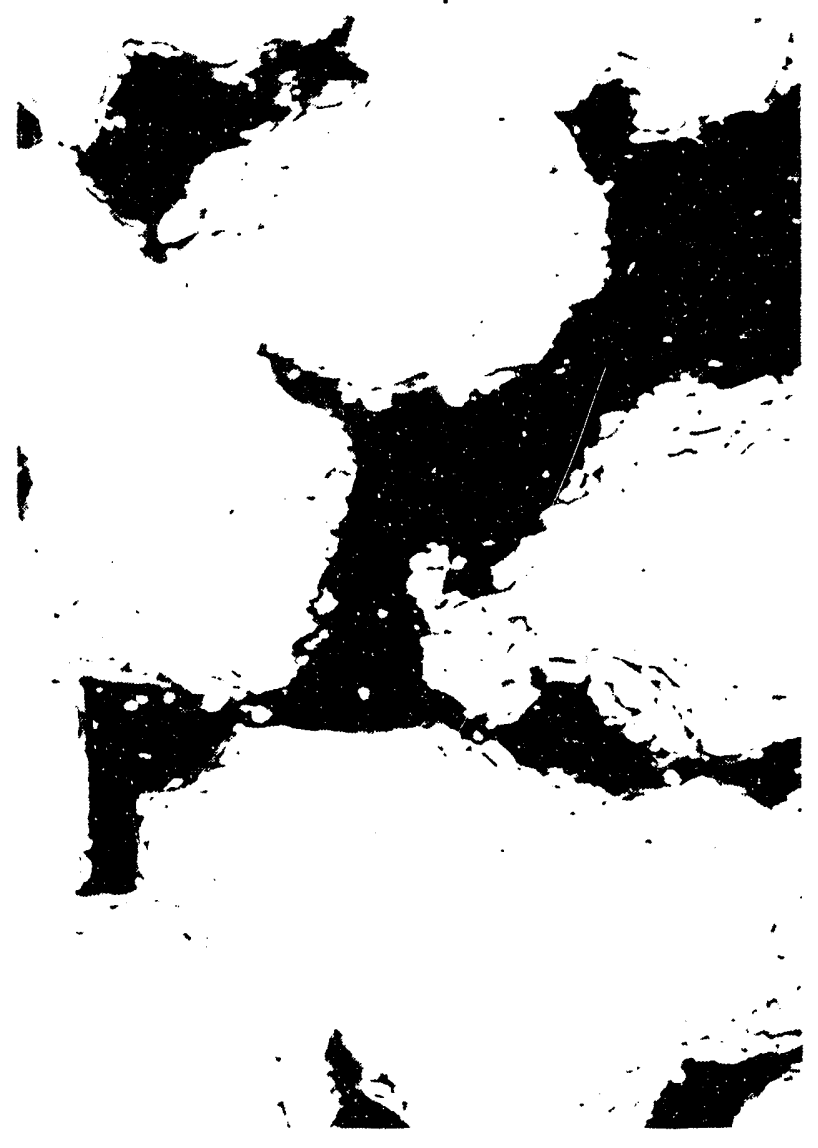

(b)

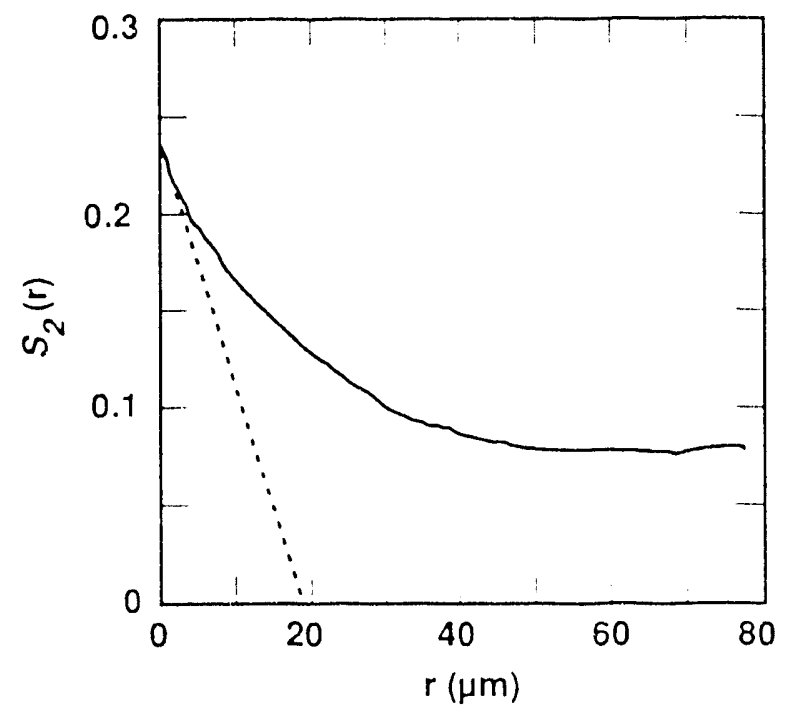

Figure 25. (a) $510 \times$ magnification SEM image and (b) related correlation function (solid curve) for Tensleep sandstone. Slope near the origin is indicated by a dashed line. 
(a) NAV

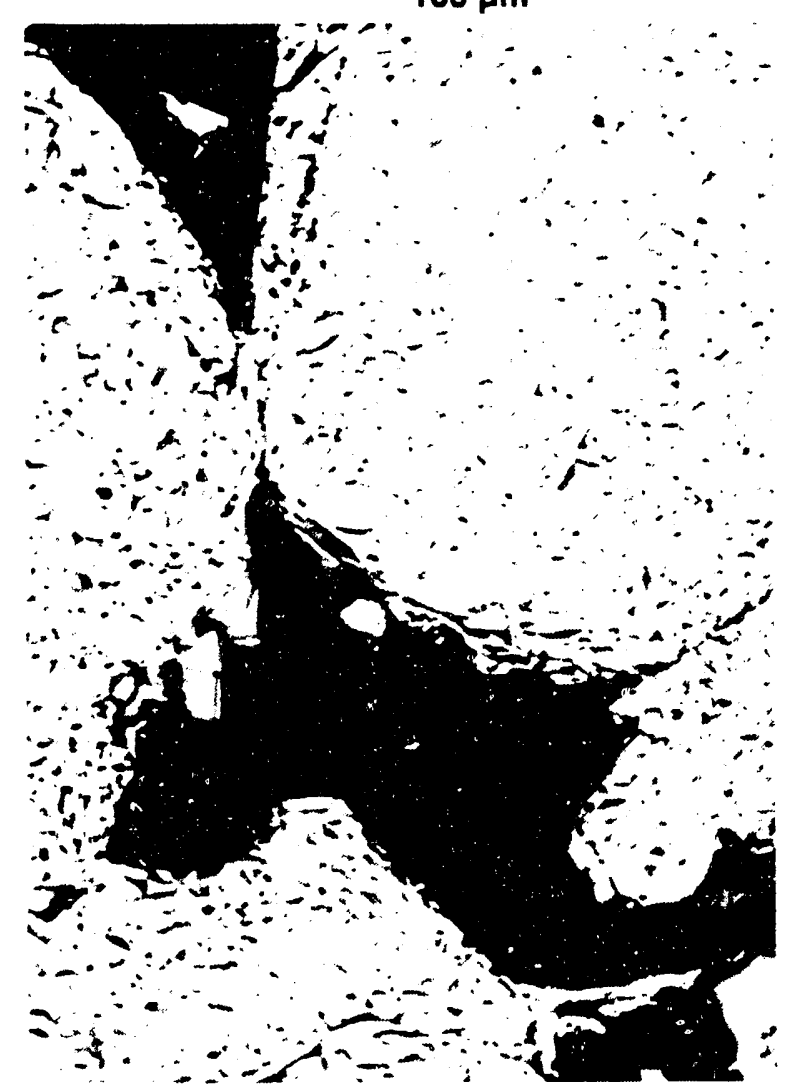

(b)

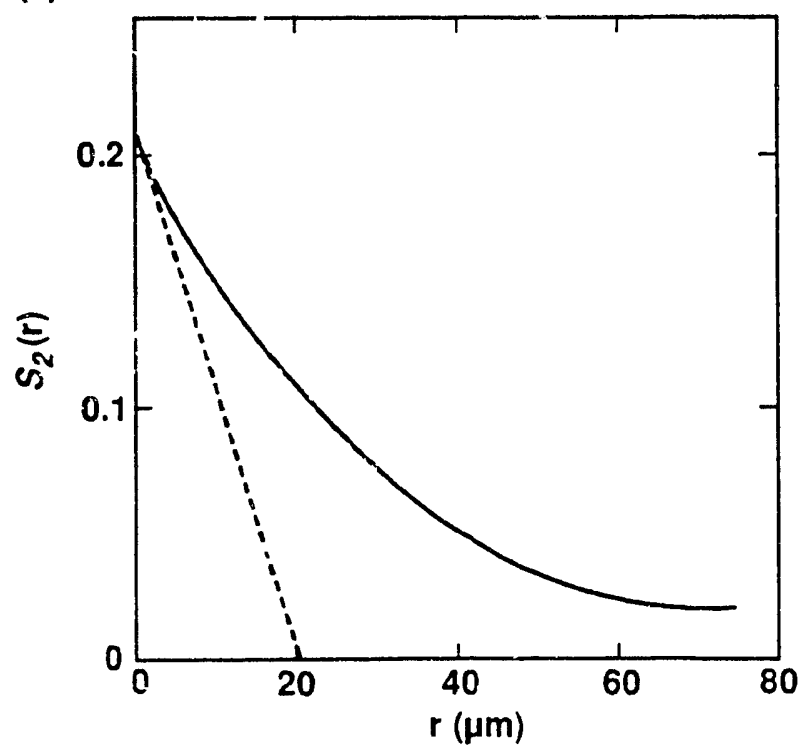

Figure 26. (a) $500 \times$ magnification SEM image and (b) related correlation function (solid curve) for Navajo sandstone. Slope near the origin is indicated by a dashed line.

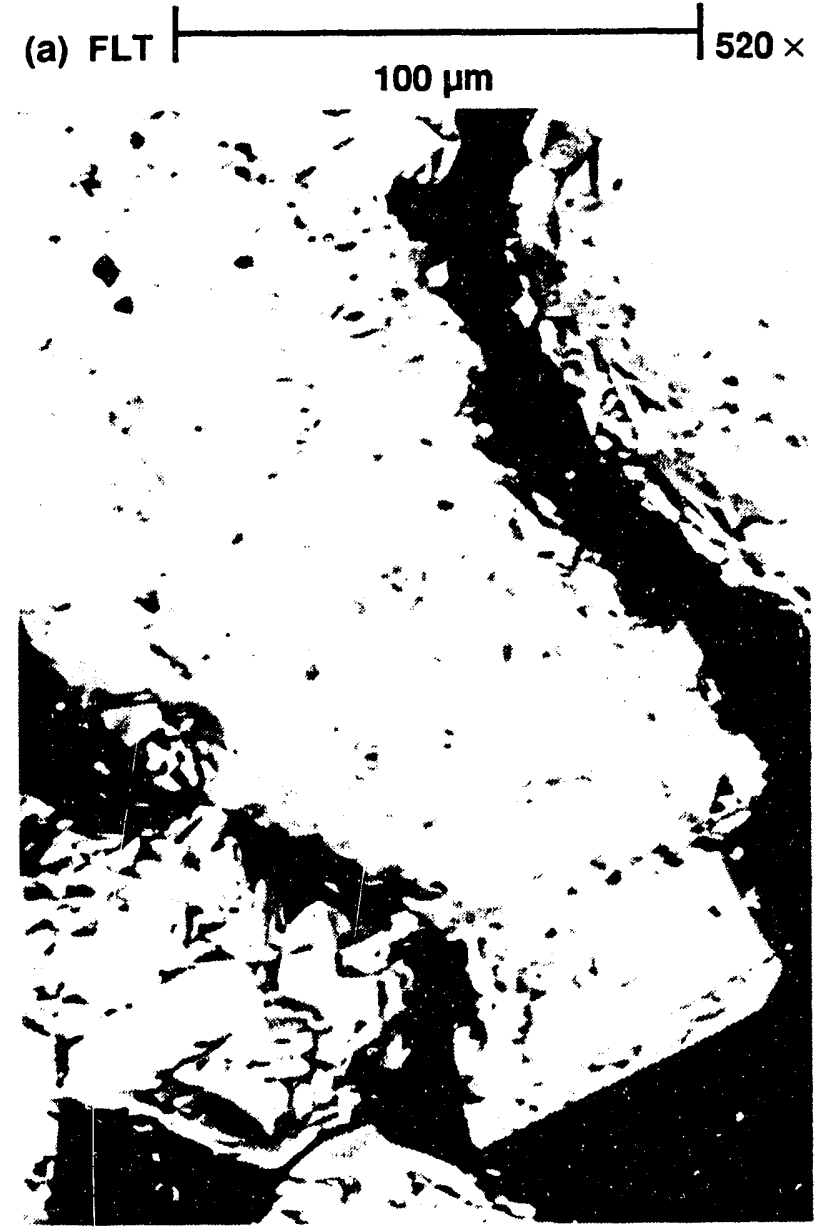

(b)

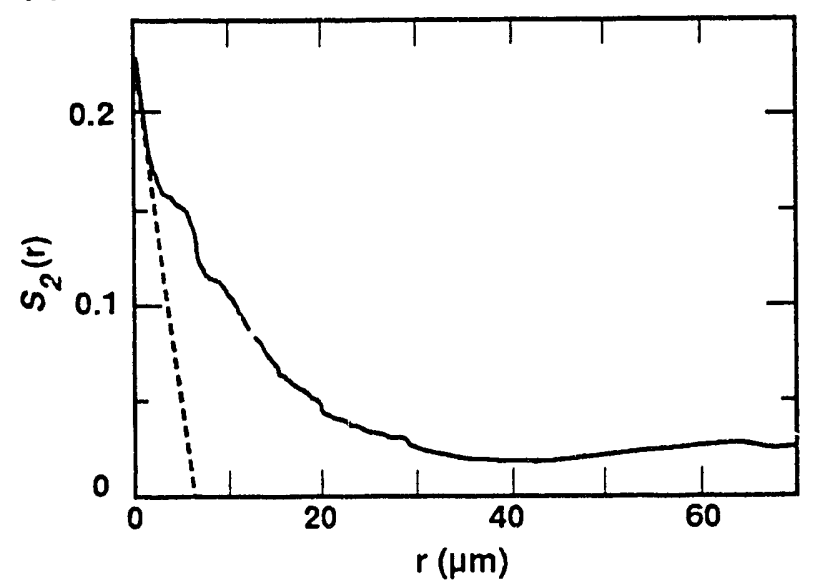

Figure 27. (a) $520 \times$ magnification SEM image and (b) related correlation function (solid curve) for Flathead sandstone. Slope near the origin is indicated by a dashed line. 


\section{Permeability}

We estimated permeability for each of the synthetic and natural sandstone samples by using computed values of porosity and image specific-surface area in Eq. (14). We used c $=2$ for all sam ples. For the natural sandstones, we used previcusly measured values of the formation factor. We estimated $F$ for the synthetic sandistone samples by using Eq. (15), with $\mathrm{m}=1.5$. Johnson et al. [1982] and Sen et al. [1981] found good agreement between this model and measured val'ses of $F$ for sintered glass beads. The predicted values fit their observed values to within a few percent for samples with porosities of about $0.27-0.34$, and the agreement was within $10-20 \%$ for samples with porosities of about $0.16-0.22$. Table 3 gives values of $F$ determined froin the $\phi_{\text {obs }}$ porosities (Table 1) and Eq. (15) for our four sintered glass-bead samples. Uncertainties in $\mathrm{F}$ are expected to be within about $5 \%$ for samples $1.2,1.4$, and $B$, and within about $20 \%$ for sample 1.1 .

For the synthetic sandstones, estimated permeabilities found using Eq. (14) are presented in Table 3, along with the measured permeabilities. [Because calculated porosities were used in Eq. (14), we have included the $\phi_{\text {calc }}$ values from Table 1 here for convenience.] Clearly, the estimated permeabilities are controlled more strongly by porosity than by specific surface. We have good agreement between calculated and observed values for all of the glass-bead samples. Using higher masnifications than $300 \times \mathrm{fcr}$ the images would have produced larger values for the image specific-surface estimates and, thus, smaller values for the estimated permeabilities and better agreement for the two lowest-porosity samples.

Table 3. Synthetic sandstone permeabilities.

\begin{tabular}{rlrcc}
\hline Sample & $\phi_{\text {calc }}$ & $F$ & \multicolumn{1}{l}{$\begin{array}{l}\mathrm{k}_{\text {calc }} \\
\text { (D) }\end{array}$} & $\begin{array}{c}\mathrm{k}_{\text {obs }} \\
\text { (D) }\end{array}$ \\
\hline B & 0.37 & 4.14 & $28 \pm 10$ & $34 \pm 5$ \\
1.4 & $0.35-0.39$ & 4.53 & $35 \pm 14$ & $32 \pm 5$ \\
1.2 & 0.33 & 6.57 & $29 \pm 3$ & $17 \pm 2$ \\
1.1 & 0.17 & 13.90 & $4 \pm 2$ & $1.3 \pm 0.2$ \\
\hline
\end{tabular}

Calculated and observed permeabilities for the natural sandstones are presented in Table 4 and are plotted in Fig. 28 together with permeabilities for the synthetic sandstones. Again, we find good agreement between observed permeabilities and the permeabilities calculated using $S_{2}$. In Table 4, the permeability appears to be controlled primarily by the porosity, as illustrated by the differences for the two Berea sandstone samples. Figure 28 shows several interesting features. First, the predicted and measured values agree generally within a factor of 2 . This indicates that for these sandstones, permeability prediction using a very simple model based on flow in an equivalent conduit is accurate, and using a more detailed model is not necessary. Moreover, Fig. 28 shows that different sandstones with similar porosities display a large range of permeabilities. That is, although porosity for these samples spans less than one order of magnitude, the permeability spans nearly three orders of magnitude. The power-law fit of permeability to porosity for our samples is also shown in Fig. 28. Comparison of Eqs. (14) and

Table 4. Permeabilities of natural sandstones.

\begin{tabular}{|c|c|c|c|c|}
\hline Sample & $\phi_{\text {calc }}$ & $\mathbf{F}$ & $\begin{array}{l}\mathbf{k}_{\text {calc }} \\
\text { (D) }\end{array}$ & $\begin{array}{l}k_{\text {obs }} \\
\text { (D) }\end{array}$ \\
\hline $1-G \# 785^{a}$ & b & $10^{c}$ & 0.424 & $0.753^{d}$ \\
\hline I-G \#775 & b & $10^{c}$ & 0.222 & $0.263^{d}$ \\
\hline Berea Berq & $0.15-0.19^{e-h}$ & $15^{i}$ & 0.153 & $0.350^{i}$ \\
\hline Frontier & $0.217 \mathrm{f}, \mathrm{h}$ & $12^{\mathrm{h}}$ & 0.179 & $0.286 \mathrm{~h}$ \\
\hline Berea BF1 & 0.1538 & 608 & 0.017 & 0.0238 \\
\hline İensleep & $0.181^{f, h}$ & $17^{\mathrm{h}}$ & 0.116 & $0.326^{h}$ \\
\hline Navajo & $0.138^{e}$ & $50 \mathrm{i}$ & 0.028 & $0.064^{h}$ \\
\hline Flathead & $0.106^{f, h}$ & $62^{h}$ & 0.010 & $0.005^{h}$ \\
\hline
\end{tabular}

a I-G signifies Ironton-Galesville sandstone.

b Not available.

c Estimated values from Berryman and Blair [1986].

d From Blair et al. [1985].

e From Coyner [1984].

f From Caruso et al. [1985].

8 From Daily and Lin [1985].

h From Wissler [1987].

i From Agrawal et al. [1991].

i Est. using $\mathrm{F}=\phi^{-2}$ [e.g., Brace et al., 1965; Wong et al., 1984]. 


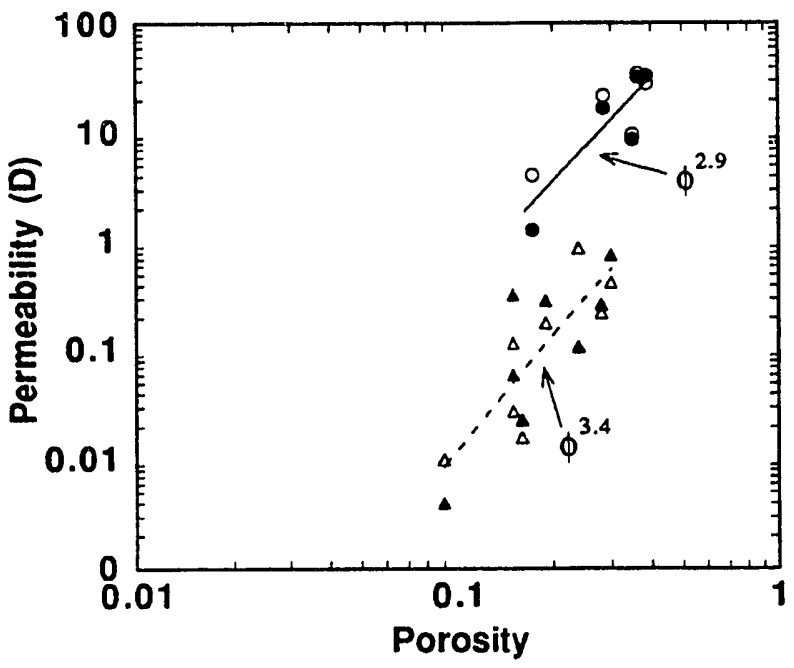

Figure 28. Variation of $\log$ permeability with $\log$ porosity. Synthetic sandstone samples (circles) and natural sandstones (triangles) are represented by solid symbols for laboratory measurements of permeability and by open symbols for permeabilities determined from correlation functions. We have included values for an additional glass-bead sample described by Berryman and Blair [1986]. Solid line gives linear fit to synthetic sandstone data; dashed line fits natural sandstone data.

(15) shows that an exponent of $2+m$ [where $m$ is defined in Eq. (15)] is expected for this fit, if $s$ does not vary rapidly with porosity. Previous studies have observed an exponent of 3 for glassbead samples [e.g., Brace, 1977; Bourbié et al., 1987]. The exponent of 3.4 found for our natural sandstones is similar to the value of 3.8 computed by Doyen [1988] for a suite of samples of Fontainebleau sandstone, and is higher than the value of about 3 observed for our synthetic sandstones. Our results indicate that the Kozeny-Carman model describes almost exactly the flow properties in synthetic sandstones and is very useful for modeling the flow properties of our natural sandstones.

\section{Relative Permeability}

We extended our analysis to cases where more than one fluid is present in the pores. Understanding of multiphase flow regimes is important for analyzing the transport of ground water and contaminants in both the saturated and partially saturated (vadose) zones. In this analysis we determine the specific surface area for the region of the pore space occupied by each fluid. We have derived a simple sum rule that provides an estimate of the interfacial area between fluid phases when total specific surface and specific surface of each fluid phase are known. The upper section of Fig. 29 shows the simplest arrangement, where one fluid completely wets the internal surface of the porous medium. A nonwetting fluid introduces a fluid-fluid interface as shown in the lower part of Fig. 29. For factors $s_{w}, s_{n w}, s_{T}$, and $s_{I}$ defined as surface areas for the respective surfaces illustrated in Fig. 29, the total surface area of the wetting fluid is

$$
\mathrm{s}_{\mathrm{W}}=\mathrm{s}_{\mathrm{T}}+\mathrm{s}_{\mathrm{I}}
$$

but $\mathrm{s}_{\mathrm{rw}}=\mathrm{s}_{\mathrm{I}}$. Adding these equations gives the general result

$$
s_{w}+s_{n w}=s_{T}+2 s_{I}
$$

[Blair and Berryman, 1991, 1992]. We applied this analysis to a sandstone sample that was sequentially imbibed with wetting and nonwetting pore-casting fluids. The rock was then sectioned and irnages of the total pore space were analyzed along with images of portions of the pore space occupied by wetting and nonwetting fluids [Agrawal et al., 1991]. Examples of raw and three-phase images are shown in Figs. 14(a) and 30 [after Blair and Berryman, 1992]. Results of the analysis have been reported in detail by Blair and Berryman [1991, 1992] and are only summarized here. Results indicate that the effective hydraulic radius of the pore space occupied by the nonwetting phase is larger than that of the total pore space. The results show qualitatively that the wetting phase dominates the specific surface while the nonwetting phase dominates the permeability, due to its higher partial saturation. This also indicates that for the imaged cross section the wetting phase is filling the dead-end pores and that much of the specific surface area is contained in those deadend pores. Additional analysis showed that the Kozeny-Carman relations and Archie's empirical laws [Archie, 1942] must be modified to account for a finite percolation threshold, and that an over-simplified theory that ignores the percolation threshold leads to unphysical values for relative permeability. 


\section{Specific surface for}

two fluids

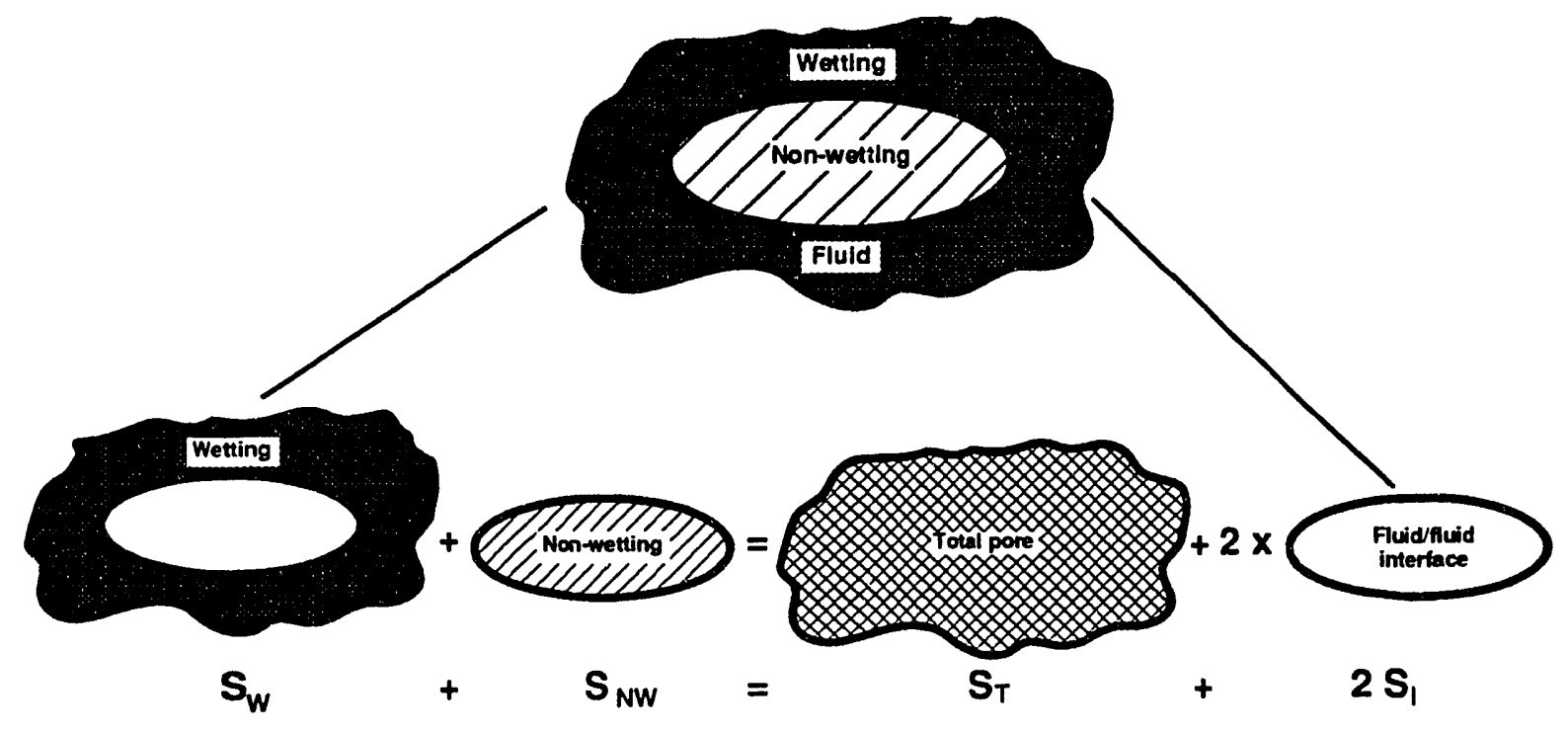

Figure 29. Conceptualization of wetting and nonwetting fluids in a pore [after Blair and Berryman, 1992].

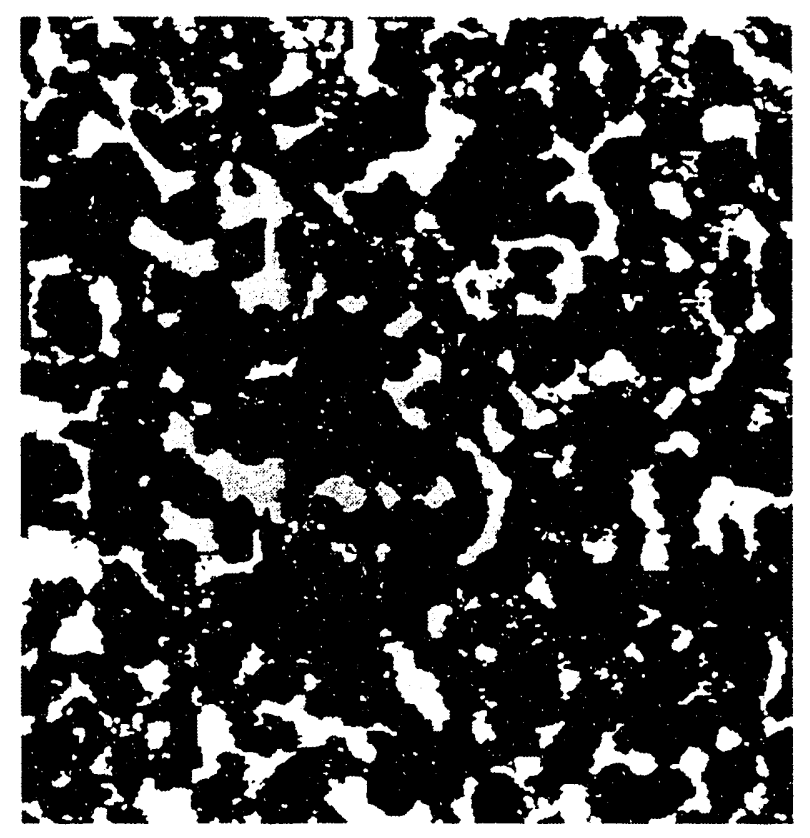

Figure 30. Three-phase image of $54 \times$ magnification Berea sandstone sample Berq. Solid grains (black) with nonwetting (grey) and wetting (white) phases [after Blair and Berryman, 1992]. 


\section{Conclusions and Discussion}

We analyzed images of cross sections of synthetic and natural sandstones using two-point spatial correlation functions. We computed porosity, pore and grain sizes, specific surface area, hydraulic diameter, and permeability for four synthetic sandstones and for eight natural sandstone samples. The computed values of porosity, effective grain size, and permeability agree well with independent measurements for all samples. We found that the two-point correlation function $S_{2}$ can be used to indicate the general morphology for natural and synthetic sandstones, and that an idealized fluid flow model described by a Kozeny-Carman relation works well for predicting permeabilities of simple sandstones such as clean quartz arenites. The permeabilty was shown to be a function of $\phi^{3}$ for our synthetic sandstones, and of $\phi^{3.4}$ for the natural sandstone samples. We find, therefore, that the image-processing methods explored in this paper are successful in obtaining reasonable estimates of physical properties related to fluid flow.

Why have we pursued these particular methods for applications to rocks? The motivations for studying image-processing methods in general and spatial correlation techniques in particular are both scientific and practical. Scientifically, details of the rock microstructure (such as the presence of cracks and fractures) are well known to play a major role in determining overall properties, such as elastic constants and fluid permeability. Yet information about actual microstructures can only be obtained by destructive methods like thin-sectioning coupled with imaging (as described in this paper), or by nondestructive methods such as volume tomographic imaging. Regardless of how images are obtained, it is essential that careful studies be made to relate measurements of physical properties to observations of real microstructures. The present work is intended to contribute to this larger scientific goal.

From a practical standpoint, measuring fluid flow properties in situ is generally very expensive. In some instances (such as poorly consolidated soils or sediments), even laboratory measurements may not be practical because intact samples are often so difficult to obtain. Image-processing techniques are now commonly available due to advances in computing. These techniques hold the promise of being able to provide reasonable and inexpensive quantitative estimates of permeability once the scientific issues concerning precision and reliability have been resolved. Further work is clearly needed to cover a wider range of interesting porous materials than have been presented here. Other applications of these methods to estimates of elastic and electrical properties will be addressed in future efforts.

\section{Acknowledgments}

The authors thank Brian Johnson for assistance in processing images of the synthetic sandstones, and Kirk Keller and Robert Sherwood for software support. This work was supported specifically by the Department of Energy's Office of Basic Energy Sciences. 


\section{References}

Adler, P. M., Porous Media: Geometry and Transports, 544 pp., Butterworth-Heinemann, Boston, 1992. Agrawal, D. L., N. G. W. Cook, and L. R. Myer, The effect of percolating structures on petrophysical properties of Berea sandstone, in Rock Mechanics as a Multidisciplinary Science, Proceedings of the 32nd U.S. Symposium on Rock Mechanics, edited by J.-C. Roegiers, pp. 345-354, Balkema, Rotterdam, 1991.

Archie, G. E., The electrical resistivity log as an aid in determining some reservoir characteristics, Trans. AIME, 146, 54-62, 1942.

Berge, P. A., B. P. Bonner, and J. G. Berryman, Seismic velocity-porosity relationships for synthetic sandstones, submitted to Geophysics, 1993.

Berryman, J. G., Computing variational bounds for flow through random aggregates of spheres, J. Comp. Phys., 52, 142-162, 1983.

Berryman, J. G.,Variational bounds on elastic constants for the penetrable sphere model, J. Phys. D: Appl. Phys., 18, 585-597, 1985a.

Berryman, J. G., Measurement of spatial correlation functions using image processing techniques, J. Appl. Phys., 57, 2374-2384, 1985 b.

Berryman, J. G., Relationship between specific surface area and spatial correlation functions for anisotropic porous media, J. Math. Phys., 28, 244-245, 1987.

Berryman, J. G., and S. C. Blair, Use of digital image analysis to estimate fluid permeability of porous materials: Application of two-point correlation functions, J. Appl. Phys., 60, 1930-1938, 1986.

Berryman, J. G., and S. C. Blair, Kozeny-Carman relations and image-processing methods for estimating Darcy's constant, J. Appl. Phys., 62, 2221-2228, 1987.

Berryman, J. G., and G. W. Milton, Normalization constraint for variational bounds on fluid permeability, J. Chem. Phys., 83, 754-760, 1985.

Berryman, J. G., and G. W. Milton, Microgeometry of random composites and porous media, J. Phys. D. Appl. Phys., 21, 87-94, 1988.

Blair, S. C., W. J. Deutsch, and P. J. Mitchell, Permeability, geochemical, and water quality tests in support of an aquifer thermal energy storage site in Minnesota, Rep. PNL-5438, 78 pp., Pacific Northwest Laboratories, Richland, Wash., 1985.

Blair, S. C., and J. G. Berryman, Estimates of permeability and relative permeability for sandstone using image analysis of cross sections, in Rock Mechanics as a Multidisciplinary Science, Proceedings of the 32nd U.S. Symposium on Rock Mechanics, edited by J.-C. Roegiers, pp. 365-374, Balkema, Rotterdam, 1991.

Blair, S. C., and J. G. Berryman, Permeability and relative permeability in rocks, in Fault Mechanics and Transport Properties of Rocks, edited by B. Evans and T. F. Wong, pp. 169-186, Academic Press, London, 1992.

Bourbié, T., O. Coussy, and B. Zinszner, Acoustics of Porous Media, 323 pp., Gulf Pub. Co., Houston, 1987.

Brace, W. F., Permeability from resistivity and pore shape, J. Geophys. Res., 82, 3343-3349, 1977.

Burns, D. R., C. H. Cheng, and R. H. Wilkens, Sandstone pore aspect ratio spectra from direct observations and velocity inversion, Technical Note, Int. J. Rock Mech. Min. Sci. E Geomech. Abstr., 27, 315-323, 1990.

Carman, P. C., Flow of Gases Through Porous Media, 182 pp., Academic Press, New York, 1956.

Caruso, L., G. Simmons, and R. Wilkens, The physical properties of a set of sandstones-Part I. The samples, Int. J. Rock Mech. Min. Sci. E Geomech. Abstr., 22, 381-392, 1985.

Corson, P., Correlation functions for predicting properties of heterogeneous materials, I. Experimental measurement of spatial correlation functions in multiphase solids, J. Appl. Phys., 45, 3159-3164, $1974 a$.

Corson, P., Correlation functions for predicting properties of heterogeneous materials, II. Empirical construction of spatial correlation functions for two-phase solids, J. Appl. Phys., 45, 3165-3170, $1974 b$.

Coyner, K. B., Effects of stress, pore pressure, and pore fluids on bulk strain, velocity, and permeability in rocks, Ph.D. thesis, Mass. Inst. of Technol., Cambridge, 1984. 
Daily, W. D., and W. Lin, Laboratory-determined transport properties of Berea sandstone, Geophysics, 50, 775-784, 1985.

Darcy, H., Les Fontaines Publiques de la Ville de Dijon, 590 pp., Victor Dalmint, Paris, 1856.

Debye, P., H. R. Anderson, Jr., and H. Brumberger, Scattering by an inhomogeneous solid. II. The correlation function and its application, J. Appl. Phys., 28, 679-683, 1957.

Doyen, P. M., Permeability, conductivity, and pore geometry of sandstone, J. Geophys. Res., 93, 7729$7740,1988$.

Dullien, F. A. L., Porous Media-Fluid Transport and Pore Structure, 396 pp., Academic Press, New York, 1979.

Ehrlich, R., S. K. Kennedy, S. J. Crabtree, and R. L. Cannon, Petrographic image analysis I. Analysis of reservoir pore complexes, J. Sed. Pet., 54, 1365-1378, 1984.

Johnson, D. L., T. J. Plona, C. Scala, F. Pasierb, and H. Kojima, Tortuosity and acoustic slow waves, Phys. Rev. Lett., 49, 1840-1844, 1982.

Koplik, J., C. Lin, and M. Vermette, Conductivity and permeability from microgeometry, J. Appl. Phys., $56,3127-3131,1984$.

Paterson, M. S., The equivalent channel model for permeability and resistivity in fluid-saturated rock-A re-appraisal, Mech. Mater., 2, 345-352, 1983.

Sen, P. N., C. Scala, and M. H. Cohen, A self-similar model for sedimentary rocks with application to the dielectric constant of fused glass beads, Geophysics, 46, 781-795, 1981.

Stanke, F., Spatial autocorrelation functions for calculations of effective propagation constants in polycrystalline materials, J. Acoust. Soc. Am., 80, 1479-1485, 1986.

Thompson, A. H., A. J. Katz, and C. E. Krohn, The microgeometry and transport properties of sedimentary rocks, Adv. Phys., 36, 625-694, 1987.

Torquato, S., and G. Stell, Microstructure of two-phase random media. I. The n-point probability functions, J. Chem. Phys., 77, 2071-2077, 1982.

Torquato, S., and G. Stell, Microstructure of two-phase random media. III. The n-point matrix probability functions for fully penetrable spheres, J. Chem. Phys., 79, 1505-1510, 1983.

Torquato, S., and G. Stell, Microstructure of two-phase random media. V. The n-point matrix probability functions for impenetrable spheres, J. Chem. Phys., 82, 980-987, 1985.

Walsh, J. B., and W. F. Brace, The effect of pressure on porosity and the transport properties of rock, J. Geophys. Res., 89, 9425-9431, 1984.

Walton, M.S., The aquifer thermal energy storage program at the University of Minnesota, in Proceedings of the International Conference on Seasonal Thermal Energy Storage and Compressed Air Energy Storage, CONF-811066, 132-140, NTIS, Springfield, Virginia, 1981.

Weissberg, H. L., Effective diffusion coefficient in porous media, J. Appl. Phys., 34, 2636-2639, 1963.

Wissler, T. M., Sandstone pore structure: a quantitative analysis of digital SEM images, Ph.D. thesis, Mass. Inst. of Technol., Cambridge, Mass., 1987.

Wong, P.-Z., J. Koplik, and J. P. Tomanic, Conductivity and permeability of rocks, Phys. Rev. B, 30, $6606-6614,1984$. 

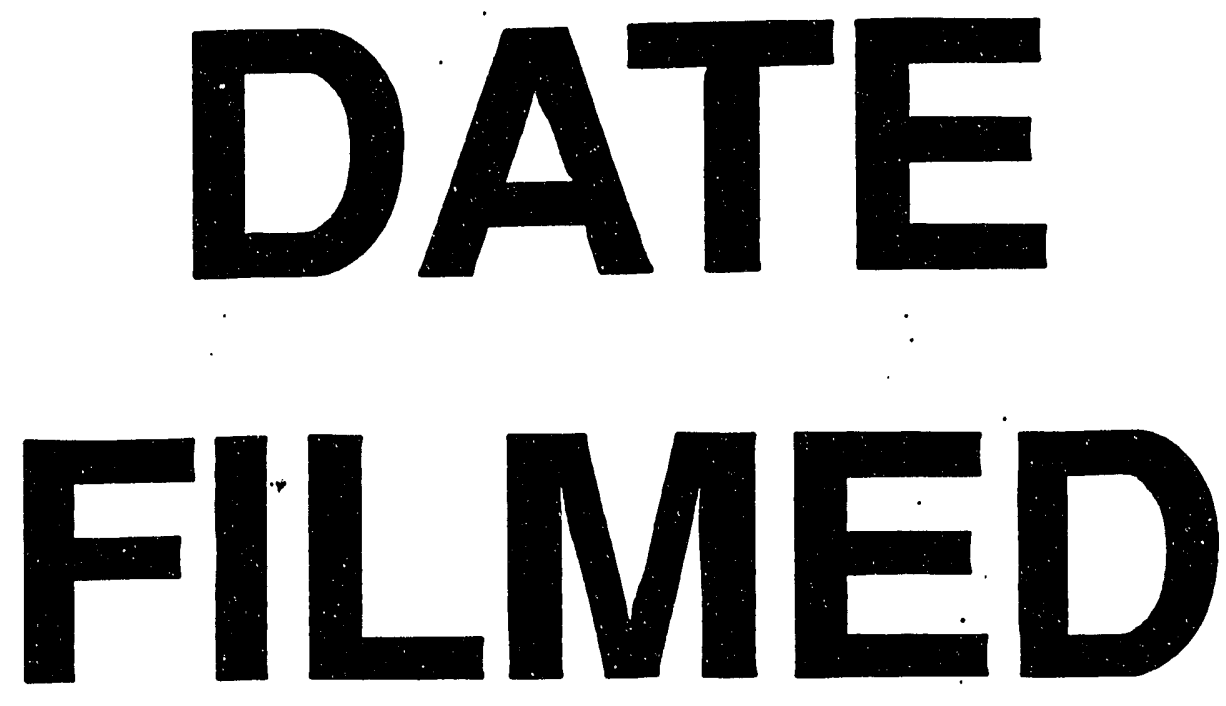

$11 / 23 / 93$
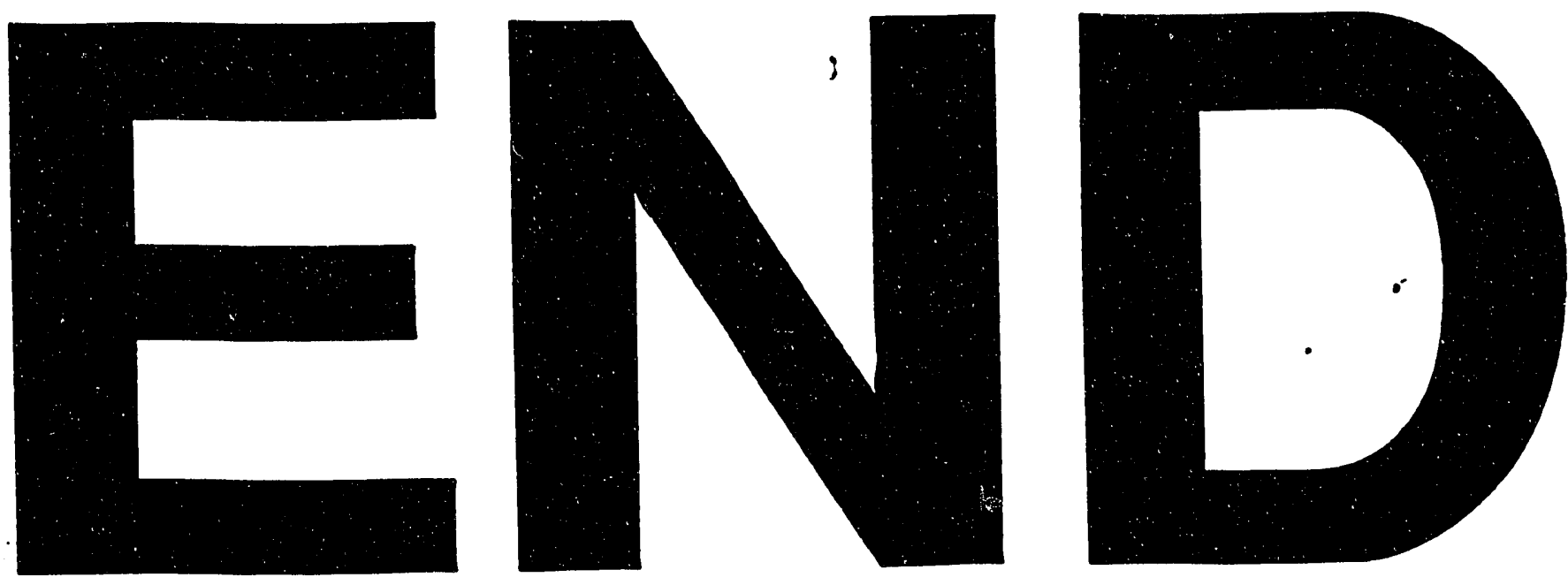
